\title{
Public Communication and Information Acquisition
}

\author{
Ryan Chahrour*
}

July 31,2012

\begin{abstract}
This paper models the tradeoff, perceived by central banks and other public actors, between providing the public with useful information and the risk of overwhelming it with excessive communication. An information authority chooses how many signals to provide regarding an aggregate state and agents respond by choosing how many signals to observe. When agents desire coordination, the number of signals they acquire may decrease in the number released. The optimal quantity of communication is positive, but does not maximize agents' acquisition of information. In contrast to a model without information choice, the authority always prefers to provide more precise signals.

JEL Classifications: E50, E58, E60, D83

Keywords: Transparency, Central Bank Communication, Monetary Policy, Global Games, Information Acquisition
\end{abstract}

\footnotetext{
*Assistant Professor, Boston College, Department of Economics. Contact at ryan.chahrour@bc.edu. Special thanks to my advisors, Stephanie Schmitt-Grohé, Ricardo Reis, Martín Uribe, and Michael Woodford for their constant guidance and support. Thank you to Ozge Akinci, Bruce Preston, Marc Giannoni, Fabià Gumbau-Brisa, Guido Lorenzoni, Alexander McQuoid, Rick Mishkin, Emi Nakamura, Kristoffer Nimark, Giovanni Olivei, Guillermo Ordoñez, Maria José Prados, Bruce Preston, Dmitriy Sergeyev, Luminita Stevens, Jón Stetinsson, Laura Veldkamp, Pierre Yared, Stephen Zeldes, seminar participants at the Midwest Macroeconomics, Canadian Economics Association, Society for Economic Dynamics, and European Economics Association annual conferences, as well as the Norges Bank, for many insightful conversations.
} 
How much should central bankers talk? Should they appoint a single speaker and provide a unified message, or allow different policymakers to discuss many topics and express dissent? Should central banks offer a detailed outlook on the economy and their objectives in setting policy? Or ought they limit themselves to very narrow statements on these topics? And, in these communications, is it better for policymakers to speak very precisely or with a degree of intentional vagueness? Central banks routinely struggle with these questions, balancing the benefits of greater transparency with the perceived risks of over-communicating. And, though communication policy varies greatly across the world's central banks, all ultimately place substantial limits on their public communication.

The priority that central banks place on crafting their communications and the preponderance of self-imposed restrictions on communication suggest that policymakers perceive tradeoffs in the choice to reveal more or less about their views on the economy. What might those tradeoffs be? The literature following Morris and Shin (2002) discusses one possibility: agents might "over-coordinate" on public information, placing more emphasis on central bank statements than is socially efficient. When public signals are imprecise, agents' over-reliance on public information may be harmful enough to warrant withholding that information altogether.

This paper provides a new account of why limits on public communication may be socially beneficial, emphasizing individuals' choices regarding the information they acquire. I propose a model in which agents' ability to coordinate their information depends on the communication policy of a benevolent information authority. The authority chooses the scope and precision of its public communications regarding an aggregate state. Scope is measured by the number of signals that the 
authority releases, while precision is measured by the variance of random noise contained in each signal. Agents choose the number of public signals to observe, and pay a cost for each. However, they cannot select precisely which of the public signals they see. Instead, the set of signals each agent observes is randomly selected from among all public signals.

In this environment, the consequences of increasing the scope of communication depend on agents' equilibrium information choices. On the one hand, if agents acquire the additional signals, increasing scope enables them to learn more about the realization of the state and, therefore, to align their actions more closely with economic fundamentals. On the other hand, if agents do not increase acquisition one-for-one with the additional signals released, the information they observe becomes more dispersed and their actions become less coordinated. When social welfare depends on coordination, this is a cost of additional communication. Which effect predominates depends on agents' information choices and, therefore, on their strategic incentives.

This model of endogenous information choice captures the tradeoff, faced by central banks and other public actors, between providing as much information as possible and ensuring common understanding among agents. Morris and Shin (2007) argue that similar considerations are important in a range of contexts, including the design of accounting rules. This motivation is often expressed colloquially as the desire to prevent confusion on the part of the public. Blinder (2007), for example, argues that too many speeches by different members of the Federal Open Market Committee (FOMC) can result in counterproductive "cacophony." In this paper, cacophony occurs when individuals each receive different parts of the central bank's overall message. This diminishes the amount of information about others' 
actions contained in each statement made by the bank and ultimately reduces the amount of information that agents choose to acquire.

Although my model nests a standard "global games" environment, I derive a number of new results regarding the consequences of communication policy. First, I find that increases in scope have a non-monotonic impact on the amount of information acquired by agents. Revelation beyond a certain critical level causes agents to decrease the amount of information they gather. This result arises because private agents with complementarities in actions find it more desirable to obtain information that they know other agents will also obtain and act on. When the authority releases signals beyond the threshold amount, an individual agent rightfully anticipates that each signal will be observed by only a fraction of other agents and will therefore be less valuable in her own decision problem. Each agent is thus less inclined to purchase public signals, which further decreases their value, and so on. As a consequence, too much information revelation results in less information acquisition, and lower welfare overall.

Second, despite the fact that releasing additional signals entails no direct cost for the authority, I find that the optimal level of scope is interior: the authority always releases a positive but finite amount of information. When agents and the authority value coordination equally, the logic behind this result is especially direct. Complete silence is never optimal, since the first bit of information released by the authority is acquired by all agents and therefore improves agents' forecast of the state without jeopardizing information coordination. Nor is releasing an unbounded number of signals optimal. The desire to create common understanding among agents ensures that the authority never chooses to release more information than agents willingly acquire in equilibrium. 
Third, I find that the optimal degree of scope entails providing substantially less information than agents would willingly acquire if it were available. This result obtains because communication policy influences the costs of coordination faced by agents. When more signals are publicly available, agents must acquire more signals in order to ensure the same degree of coordination amongst themselves. If the authority desires coordination, then it chooses to limit this cost, even though agents would pay for and acquire extra signals if they were released by the authority.

Lastly, I find that the authority always chooses to provide the most precise signals possible. A literature characterizes cases where increases in the precision of a public signal are socially harmful (Morris and Shin, 2002; Hellwig, 2005; Angeletos and Pavan, 2007a,b). My model nests these cases, but the addition of information choice changes the findings: an authority that is free to adjust the scope of its communication always prefers to use this margin of adjustment rather than reduce the precision of its signals. Thus, although the model rationalizes limits to the extent of a central bank's communication, it does not support a policy of "constructive ambiguity." The distinction between scope and precision offers one resolution to the incongruity of central bankers' commitment to transparency and their practice of limiting communication.

My conclusions both complement and contrast with those from the earlier literature on the social value of public information, including Morris and Shin (2002) and Angeletos and Pavan (2007a). Like Morris and Shin (2002), I find that more public information is potentially harmful. However, the mechanism behind this result is very different. In my model, increases in scope may be harmful because they prevent agents from coordinating their information. In the earlier literature, increasing the precision of a public signal leads to over-coordination in actions. To 
obtain this result, these studies require a misalignment between the preferences of agents and those of the social planner. However, Hellwig (2005) and Roca (2010) show there is no such misalignment in two simple new-Keynesian models with micro-founded social objectives, and therefore no reason to limit the precision of public statements in their context. In contrast, my conclusions regarding limited scope do not rely on any preference misalignment and therefore would apply in their examples.

Woodford (2005) and Svensson (2006) further argue that, even if one accepts the assumption of misaligned preferences, the parameter values under which increases in precision may be harmful are implausible: public information would need to be of extremely low quality in order for the release of a public signal to be harmful. By contrast, the results in this paper are robust to a wide range of parameters. Finally, the results of Morris and Shin (2002) imply that the authority should reveal as much information as possible, or nothing at all. In my model, optimal scope is always positive, finite, and in most instances varies continuously as a function of the parameters in the model.

This paper builds on a growing literature modeling the endogenous acquisition of information, including the foundational contributions of Sims (2003) and Reis (2006). Hellwig and Veldkamp (2009) argue that multiple equilibria may become prevalent in models of information acquisition when agents face strategic complementarities in their actions. Multiplicity arises because a signal that is observed by other agents is informative about their actions and, thus, is discretely more valuable than a signal that is not observed by others. Myatt and Wallace (2010) overcome this multiplicity by assuming that agents choose the precision with which they observe a given signal. The baseline version of my model achieves uniqueness 
by ensuring that all signals are equally informative about aggregate actions. I later extend the model of information acquisition to nest both my baseline model and that of Hellwig and Veldkamp (2009). I show that this general approach accommodates both cases with extensive multiplicity and cases of equilibrium uniqueness, despite the presence of complementarities. The model with multiple equilibria is still policy-relevant: a policymaker who cannot predict precisely which equilibrium will arise may still wish to influence the set of possible equilibria.

The few studies of communication with endogenous information choice come to mixed conclusions about the benefits of transparency. These studies typically explore models in which private sector actors pay a cost to acquire private information but receive an information authority's signals for free. Wong (2008),Ueda (2010), Kool et al. (2011) all argue that providing more precise public information may be harmful, as it crowds out agents' acquisition of private information, making aggregate actions less informative to the policy maker. In contrast, Colombo and Femminis (2008) focus on the real resource savings generated when agents substitute away from private information acquisition towards a more precise public signal and find a "pro-transparency" result. Llosa and Venkateswaran (2012) and Colombo et al. (2012) characterize inefficiency in private information acquisition in general environments, the later demonstrating circumstances where the Morris and Shin (2002) result is again reversed. In contrast, this paper emphasizes the costs of acquiring public messages, and explores implications for both the quantity and the precision of public communication.

Reis (2010) studies the question of when a policy maker should release its own private information about a coming change in regime, under a version of rational inattention. Among other results, he finds that announcements made too early 
may be ignored by agents, and that optimal communication policy takes this into account. Reis (2010) and this paper share a common theme: good communication policy requires that public institutions take care to ensure agents pay the right amount of "attention" to their message.

This paper proceeds as follows. Section 1 details agents' preferences and the model of information acquisition. Section 2 characterizes equilibrium in the model, and describes the consequences of communication policy for information choice. Sections 3 derives implications for optimal communication policy when agents' preferences are aligned with those of the social planner and when agents and the planner value coordination to differing degrees. Section 4 extends the model to allow agents to direct their search towards particular signals, and studies the consequences for determinacy in the model. Section 5 concludes.

\section{Model}

The model is a static "global games" model, with endogenous information acquisition. The preference structure follows that of Angeletos and Pavan (2007a) and nests Morris and Shin (2002). Prior to choosing their actions, agents and the authority choose information in a two-stage game, in which the authority is a Stackelberg leader. In this section, I first detail agents' preferences and then describe the information game.

\section{$1.1 \quad$ Preferences}

The economy consists of a continuum of expected utility-maximizing agents, indexed by $i \in[0,1]$, and an information authority, denoted G. Each agent chooses 
an action, $p^{i} \in \mathbb{R}$, given their information. Agent $i$ 's preferences (exclusive of information costs, which are introduced below) are given as minus a quadratic loss function, so that

$$
-u^{i}\left(p^{i} \mid \theta, p\right)=(1-\alpha)\left(p^{i}-\theta\right)^{2}+\alpha\left(p^{i}-p\right)^{2}
$$

where $\theta$ represents an aggregate economic fundamental relevant to all agents and $p \equiv \int_{0}^{1} p^{j} d j$ gives the cross-sectional average action across agents. The degree of strategic complementarity in the agent's choice is measured by $\alpha \in[0,1) .{ }^{1}$ Under these preferences, agent $i$ 's optimal response function given her information is

$$
p^{i *}\left(\mathcal{I}^{i}\right)=(1-\alpha) E^{i}[\theta]+\alpha E^{i}[p]
$$

where the notation $E^{i}[x]$ denotes expectations conditional on agent $i$ 's information set, $\mathcal{I}^{i}$.

Social welfare in the economy is measured by

$$
-u^{G}\left(\left\{p^{i}\right\}, \theta\right)=\left(1-\alpha^{\star}\right) \int_{0}^{1}\left(p^{i}-\theta\right)^{2} d i+\alpha^{\star} \int_{0}^{1}\left(p^{i}-p\right)^{2} d i
$$

In equation (1.3), the parameter $\alpha^{\star} \in[0,1]$ measures the degree of complementarity from the social planner's perspective, which may be different from that perceived by agents.

The definition of the dispersion-loss term used here, $\int_{0}^{1}\left(p^{i}-p\right)^{2} d i$, follows Angeletos and Pavan (2007a). Although it differs slightly from the definition used in

\footnotetext{
${ }^{1}$ Avoiding the case $\alpha=1$ ensures uniqueness in agents' equilibrium actions, given their information.
} 
Morris and Shin (2002), none of their computations depend on this choice. The alternative definitions, however, do have consequences whenever $\alpha^{\star}>0$. When $\alpha=\alpha^{\star}$, this choice of preferences ensures agreement between the agents' and planner's preferred actions given information (Angeletos and Pavan, 2007a). In proposition 3, I establish that whenever $\alpha=\alpha^{\star}$ agents' individual information choices are socially optimal, as well. Consequently, when $\alpha=\alpha^{\star}$, I will say that the agents' and planner's preferences are "aligned."

Many models can be cast in or approximated by the preference structure described above. Hellwig (2005) and Roca (2010) derive such preferences in pricing models with imperfect competition and monetary policy, and show that coordination in prices is desirable at both the individual and social levels $\left(\alpha \approx \alpha^{\star}\right)$. Because of its relevance to central banking, this example motivates my initial focus on the case of aligned preferences. Other examples in which coordination is both individually and socially desirable $\left(\alpha \leq \alpha^{\star}\right)$ include the model of strategic investment decisions by Angeletos and Pavan (2004) and the model of political leadership by Dewan and Myatt (2008). In other cases, such as the asset market beauty contest

described by Allen et al. (2006) or the model of corporate board decision-making by Malenko (2011), agents desire coordination even though it brings no social benefit $\left(\alpha>\alpha^{\star}=0\right)$.

\subsection{The Communication Game}

Before the realization of random variables, the information authority and agents choose information in a two stage game. In the first stage, the information authority selects its communication policy, which consists of selecting the number of signals to release (scope) as well as their precision. In the second stage, each 


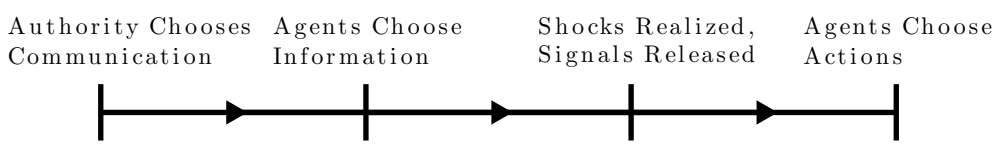

Figure 1: Timeline of the Communication Game

agent $i$ chooses her individual information allocation, taking as given the information choice of other agents as well as the communication policy of the authority. Once communication policy and information allocations are made, uncertainty is realized, signals are observed, and agents choose their actions in an individually optimal manner given their information. Figure 1 summarizes the sequence events in the model.

\subsubsection{Authority's Communication Policy}

The aggregate state $\theta$ is normally distributed with mean zero and variance $\sigma_{\theta}^{2}$ normalized to one. Its realization is known perfectly to the information authority. The authority can choose to share its information with the public by costlessly providing one or more signals, indexed by $l=1, \ldots, n$, of the form

$$
g_{l}=\theta+\eta_{l}
$$

The error in each signal is assumed to be gaussian white noise, i.i.d. across signals, with variance $\sigma_{\eta}^{2} \geq \sigma_{\eta}^{2}$. Since I later show that the authority always selects the most precise (lowest $\sigma_{\eta}^{2}$ ) signals possible, I initially take this value as exogenous. The only remaining choice of the authority is scope, measured by $n$, the number of signals it wishes to make available for public observation. The assumption that the authority knows the state exactly is for expositional convenience only. I relax 
this assumption and study the consequences in section 3.2.

Why is scope an interesting dimension of communication? The idea behind this formulation is that providing more information about a complex concept (e.g. "the state of economy") requires more communication on behalf of the authority or central bank. To internalize the authority's communication is costly to agents; they cannot make use of additional communication unless they expend the resources needed to process it. This gives agents' incentives an important new role in shaping the consequences of public communication. This view of communication fuses the main insights of the literature on information choice (that agents influence the information embodied in their actions) and the literature on public information (that policy-makers influence the informational environment faced by agents.)

Because of the emphasis on costly information acquisition, the scope concept maps most naturally into current debates about how much communication to undertake. For example, should the central bank provide the public with the details of its internal debates about policy, as is the case for the Bank of England, or with a more unified view, as the European Central Bank currently does? Should it provide forecasts of the policy rate, as a number of inflation-targeting banks do? Should it describe the details of its models and forecasting assumptions? In contrast, changes in the precision of signals are free and are necessarily "absorbed" as greater information flows to the private sector. This dimension of communication maps more naturally into the debate about "constructive ambiguity," the question of whether intentional obfuscation in a given public statement might be warranted. 


\subsubsection{Agent's Information Choice}

Agents are Bayesian, and share a model-consistent prior on the state. In addition to any public information they choose to acquire, agents are exogenously endowed with a private signal

$$
r^{i}=\theta+\xi^{i}
$$

where $\xi^{i}$ is i.i.d. $N\left(0, \sigma_{\xi}^{2}\right)$ across agents. ${ }^{2}$

Given the authority's communication policy, each agent must choose how many of the public signals to observe, $k \leq n$, taking as given the information choices of other agents. To make these observations, agents pay a utility cost of $c(k)=\lambda k$. I assume that agent $i$ cannot select precisely which of the $n$ signals to observe, and instead observes a subset drawn randomly without replacement from among the signals released by the authority. This assumption is important; I discuss its relevance below and show how it may be substantially relaxed in section 4 .

At the time of her actions, I assume agent $i$ knows the realizations of $k$ out of the $n$ public signals and can associate each of her observations with the index of a particular signal, $l .^{3}$ With a slight abuse of notation, I will say $g_{l} \in \mathcal{I}^{i}$ whenever $g_{l}$ is among the signals observed by agent $i$.

To choose her action, agent $i$ must forecast both the state, $\theta$, and the average action, $p .{ }^{4}$ As I demonstrate in the appendix, the later requires forming expec-

\footnotetext{
${ }^{2}$ The assumption of exogenous private information is not without loss of generality. However, as long as the cost-per-unit-precision of private information exceeds that of public information, agents will always exhaust all available public signals before considering the acquisition of additional private information. In this case, the subsequent theorems follow with very few modifications.

${ }^{3} \mathrm{An}$ alternative assumption is that the agent cannot verify ex-post which of the signals she has observed. Since all signals are selected with equal probability, however, this choice is inconsequential.

${ }^{4}$ I prevent agents from observing a signal related to $p$ directly, and therefore abstract from any consideration of the information aggregate actions (e.g. prices) convey to agents. This issue
} 
tations about both private signals of other agents and about those public signals observed by other agents but not by agent $i$. These expectations can be written in terms of the conditional expectation of $\theta$. This expectation is given by

$$
E\left[\theta \mid \mathcal{I}^{i}\right]=\gamma_{1} \sum_{g_{l} \in \mathcal{I}^{i}} g_{l}+\gamma_{2} r^{i}
$$

where $\gamma_{1}=\left(k+\sigma_{\eta}^{2}\left(1+\frac{1}{\sigma_{\xi}^{2}}\right)\right)^{-1}$ and $\gamma_{2}=\frac{\sigma_{\eta}^{2}}{\sigma_{\xi}^{2}} \gamma_{1}$. The agent's inference problem is detailed in appendix A.1.

The assumption that agents cannot influence which of the $n$ signals to observe is important. This assumption, and the assumption of Hellwig and Veldkamp (2009) that agents can perfectly select their preferred signals, are each special cases of a general model, in which agents search across signals with a limited ability to direct that search towards their most preferred signals. The current setup is analogous to an environment of undirected search, since agents cannot influence which of the public signals they observe. Permitting perfectly directed search, with no corresponding cost, introduces a large multiplicity of equilibria into the model, for precisely the reasons described by Hellwig and Veldkamp (2009). In section 4 , I extend the model to allow agents to choose the probability of observing each individual signal, nesting both of the above cases, and establish conditions on the cost of information that are sufficient to ensure that search is undirected in equilibrium.

Costly coordination (via costly directed search) is one plausible micro-foundation for the assumption that agents receive different messages from the authority, but the model could be extended to incorporate others as well. Agents might face is studied by Amador and Weill (2010) and Vives (2010), among others. 
individual-specific precisions or costs of acquiring various signals, reflecting the idea that individuals specialize in certain topics or find certain speakers more intelligible. Alternatively, one might view such dispersion as the result of information aggregation: real agents must acquire information about a great deal of relevant variables in order to make many different decisions and almost always gather multiple kinds of information from a single source. If each agent's choice of aggregator is driven primarily by idiosyncratic considerations, then agents again may fail to coordinate on precisely which signals they observe.

\subsection{Definitions}

In this section, I define concepts of equilibrium and efficiency, taking communication policy as given. Let $U^{i}\left(k, p^{i}\left(\mathcal{I}^{i}\right) ; p(G)\right) \equiv E\left[u^{i}\right]-c(k)$ be the unconditional expected utility of agent $i$ as a function of her information allocation, $k$, and her action rule $p^{i}\left(\mathcal{I}^{i}\right)$, and the aggregate mapping of the state and authority's signals to aggregate actions, $p(G)$. Similarly, define $U^{G}\left(k, p^{i}\left(\mathcal{I}^{i}\right) ; p(G)\right) \equiv E\left[u^{G}\right]-c(k)$ to be the unconditional expected value of aggregate social welfare. ${ }^{5}$

Definition 1. Given communication policy, $\left\{n, \sigma_{\eta}^{2}\right\}$, a pure strategy symmetric equilibrium consists of an information allocation and an action rule mapping information to actions, $\left\{k^{*}, p^{i *}\left(\mathcal{I}^{i}\right)\right\}$, such that

1. Each agent's choice of information and actions maximize expected utility, taking other agents' actions as given:

$$
\left\{k^{*}, p^{i *}\left(\mathcal{I}^{i}\right)\right\}=\underset{k, p^{i}\left(\mathcal{I}^{i}\right)}{\operatorname{argmax}} U^{i}\left(k, p^{i}\left(\mathcal{I}^{i}\right) ; p(G)\right) \quad \text { subject to } \quad k \leq n .
$$

\footnotetext{
${ }^{5}$ This notation reflects a restriction to symmetric information allocations and action rules.
} 
2. The average action is given by $p(G)=\int_{0}^{1} p^{i^{*}}\left(\mathcal{I}^{i}\right) d i$.

The restriction to pure strategy equilibria simplifies notation, and will not exclude any equilibria under assumption 1, which I present shortly and maintain throughout the paper.

I now define a benchmark that will be helpful in evaluating the efficiency of the decentralized equilibria in the model.

Definition 2. Given a communication policy, $\left\{n, \sigma_{\eta}^{2}\right\}$, the socially optimal symmetric information-action plan consists of an $i$-common information allocation and an action rule, $\left\{k^{\bullet}, p^{\bullet}\left(\mathcal{I}^{i}\right)\right\}$, which satisfy

$$
\begin{gathered}
\left\{k^{\bullet}, p^{i}\left(\mathcal{I}^{i}\right)\right\}=\underset{k, p^{i}\left(\mathcal{I}^{i}\right)}{\operatorname{argmax}} U^{G}\left(k, p^{i}\left(\mathcal{I}^{i}\right) ; p(G)\right) \quad \text { subject to } \\
k \leq n ; \\
p(G)=\int_{0}^{1} p^{i}\left(\mathcal{I}^{i}\right) d i
\end{gathered}
$$

The definition of the planner's information-action plan corresponds to the efficiency criterion used in Angeletos and Pavan (2007a), in that it considers efficiency given the constraint on information. This definition extends theirs, however, by considering efficiency over a range of potential information allocations, rather than considering actions for a fixed information structure.

I will later establish that, when $\alpha=\alpha^{\star}$, a unique equilibrium satisfying definition 1 will always correspond to the socially optimal information-action plan. In contrast, when $\alpha \neq \alpha^{\star}$, either actions, information acquisition, or both, may be inefficient. 


\section{Equilibrium and Efficiency}

The model is solved by optimizing sequentially, first choosing actions taking information as given, and then choosing the information allocation assuming that the use of information is individually optimal.

\subsection{Exogenous Information}

In this section, I solve for the equilibria of the model taking the information choice of agents as given. I restrict myself to the space of linear equilibria, such that the mapping from signals to the aggregate action can be represented by a linear rule

$$
p=\psi_{1}^{*} \sum_{l=1}^{n} g_{l}+\psi_{2}^{*} \theta
$$

In appendix A.2, I use a method of undetermined coefficients and iterate on agent's optimal action given in equation (1.2) to solve for $\psi_{1}^{*}$ and $\psi_{2}^{*}$. Conditional on observing a particular public signal, individual $i$ 's response to that signal is then given by

$$
\psi_{1}^{i *}=\frac{n}{k} \psi_{1}^{*}=\left(k+\left(1-\alpha \frac{k}{n}\right) \sigma_{\eta}^{2}\left(\frac{1}{1-\alpha}+\frac{1}{\sigma_{\xi}^{2}}\right)\right)^{-1}
$$

Compare now the expression for $\psi_{1}^{i^{*}}$ in equation (2.2) with the optimal weight of inference on the corresponding observation, $\gamma_{1}=\left(k+\sigma_{\eta}^{2}\left(1+\frac{1}{\sigma_{\xi}^{2}}\right)\right)^{-1}$. Clearly, $\psi_{1}^{i *}=\gamma_{1}$ whenever agents have no strategic complementarities and they acquire all of the public signals $(\alpha=0$ and $k=n)$. Further inspection reveals that $\psi_{1}^{i *}>\gamma_{1}$ whenever agents acquires all signals but $\alpha$ is positive. Conversely, in this case, $\psi_{2}^{*}<\gamma_{2}$. Thus, agents "overweight" the public signal relative to the Bayesian 
weights. ${ }^{6}$ This is the effect highlighted by Morris and Shin (2002): agents' desire to coordinate actions causes them to respond more strongly to public signals than they otherwise would.

\subsection{Morris and Shin (2002) Result}

When $k=n=1$ and $\alpha^{\star}=0$, the model nests that of Morris and Shin (2002). The fundamental result of that paper and the subsequent literature (e.g. Angeletos and Pavan, 2007a), is that social welfare may be decreasing in the precision of public information when agents place inefficiently high weight on coordination. ${ }^{7}$ This basic result is captured in proposition 1, which is proved in appendix A.3.

Proposition 1. Suppose $n=k=1$ and $\alpha^{\star}=0$. Then social welfare is decreasing in $\frac{1}{\sigma_{\eta}^{2}}$ if and only if

$$
(2 \alpha-1)(1-\alpha)>\sigma_{\xi}^{2}\left(1+\frac{1}{\sigma_{\eta}^{2}}\right) .
$$

As pointed out by Svensson (2006), the prerequisites of condition (2.3) are stringent, for it requires both that $\alpha>\frac{1}{2}$ and that private information is much more precise than public information. Explicitly including agents' common prior highlights an additional requirement: private information must also be very precise in an absolute sense. If this requirement is not met, then agents do not update their beliefs much in response to either signal, and the resulting inefficiency in actions is too small to offset the benefits of making the public signal more precise.

An important implication of proposition 1 is that an authority choosing the

\footnotetext{
${ }^{6}$ Note, however, that when $\frac{k}{n}$ is sufficiently small, it may be that $\psi_{1}^{i *}<\gamma_{1}$. This occurs because the common prior plays a role that is identical to a common public signal. For small enough $\frac{k}{n}$, agents overweight the prior relative to all other information.

${ }^{7}$ Conversely, when agents underweight coordination, increases in the precision of private information may be harmful.
} 
precision of its signal subject to an upper bound always chooses to provide the most precise signal possible or no signal at all. To see this, note that whenever the condition in (2.3) holds for a particular value $\underline{\sigma_{\eta}^{2}}$, it must also hold for values of $\sigma_{\eta}^{2}>\sigma_{\eta}^{2}$. In such cases the authority selects the variance of its signal to be arbitrarily large: the authority provides no information at all to the public. I follow Morris and Shin (2002) in calling this a "bang-bang" result.

\subsection{Endogenous Information}

In this section, I solve for the equilibrium information choice, assuming that information is used in the equilibrium manner. In appendix A.4, I derive that the loss function of agent $i$ as univariate function of $k$, taking the information choice and equilibrium actions of other agents as given. I now turn to deriving the equilibrium choice of $k$.

\subsubsection{Continuous Information}

For analytical simplicity, I focus on a version of the model in which the information choices of the authority and agents can each be described by continuous parameters $\hat{k}$ and $\hat{n}$, rather than the integers $k$ and $n$. To transform the model into its continuous analogue, I divide each "unit" of information provided by the authority into a set of sub-units, and so on, ad infinitum. The details of this transformation are given in appendix A.4.1, where I show that the discrete expressions for the equilibrium coefficients and social welfare can be mapped into isomorphic expressions with $\hat{n}$ and $\hat{k}$ as continuous choice variables. Accordingly, I drop this distinction in the main text and simply treat $n$ and $k$ as continuous choices. 


\subsubsection{Equilibrium with Information Choice}

Assumption 1 is maintained throughout the remainder of the paper and is necessary to ensure that agents choose to acquire a non-zero quantity of information. Assumption 1. Information costs are not too high:

$$
\lambda<\frac{1}{\sigma_{\eta}^{2}\left(\frac{1}{1-\alpha}+\frac{1}{\sigma_{\xi}^{2}}\right)^{2}} .
$$

Letting $\tau \equiv\left(\frac{1}{1-\alpha}+\frac{1}{\sigma_{\xi}^{2}}\right)$, assumption 1 reduces to $\lambda<\left(\hat{\sigma}_{\eta} \tau\right)^{-2}$. The assumption states that the costs of information should not be too high, relative to the precision of public information. ${ }^{8}$ When complementarities are high, the assumption requires a relatively low cost of information. This is natural, since in this case agents are more concerned with coordination than with the value of the state per se. This logic is especially straightforward when agents have no private information. In this case, agents can coordinate perfectly if no one acquires any information about the state.

Proposition 2, proved in appendix A.4, establishes the characteristics of equilibrium information choice in the model.

Proposition 2. Let $\widehat{n} \equiv\left(\frac{\sigma_{\eta}^{2}}{\lambda}\right)^{\frac{1}{2}}-(1-\alpha) \sigma_{\eta}^{2} \tau$. Then, the equilibrium information allocation is unique and is given by

$$
k^{*}= \begin{cases}n & \text { if } n \leq \widehat{n} \\ \ddot{k}(n) & \text { otherwise, }\end{cases}
$$

${ }^{8}$ Of course, if the cost of information is so high that agents never acquire any information in equilibrium, then the choice of scope is irrelevant. 
where

$$
\ddot{k}(n)=\frac{\left(\frac{\sigma_{\eta}^{2}}{\lambda}\right)^{\frac{1}{2}}-\sigma_{\eta}^{2} \tau}{1-\frac{\alpha}{n} \sigma_{\eta}^{2} \tau} .
$$

The contrast between the uniqueness result here and the pervasive multiplicity in Hellwig and Veldkamp (2009) stems from the fact that, in their paper, agents may freely coordinate on the signals they wish to observe. From an agent's perspective, this creates a discontinuity in value between signals that are already observed by other agents (and therefore contain information about their actions as well as the state) and those that are not observed by others (and therefore only contain information about the state.) This discontinuity generates a range of values of information acquisition in which agents have no incentive to acquire either more or less information, given the information choices of other agents. ${ }^{9}$

Uniqueness in this case follows from the assumption that agents must randomize in selecting the signals that they observe. As a result, all signals are observed with equal probability by other agents and an agent deciding whether to observe an additional signal knows that all signals are equally informative about others' actions. Decreasing returns to additional observations arise only because agents update their beliefs less in response to new information once they are already observing a great deal of information.

\subsection{Scope and Information Acquisition}

How does communication policy affect equilibrium information acquisition? Corollary 1 establishes the important result that, for levels of scope beyond the

\footnotetext{
${ }^{9} \mathrm{I}$ am ignoring a different sort of multiplicity that occurs because agents could select to observe any set of $k$ signals. Because I assume that signals are a priori identical, the set of equilibria, each focusing on a different set of $k$ signals, are equivalent from a welfare perspective.
} 
critical value $\widehat{n}$, an increase in the scope of communication actually decreases the amount of information acquired by agents.

Corollary 1. Suppose that $n>\widehat{n}$. Then information acquisition is decreasing in scope:

$$
\frac{\partial \ddot{k}(n)}{\partial n}<0 .
$$

Proof of Corollary 1. To see this result, compute the derivative of $\ddot{k}(n)$ :

$$
\frac{\partial \ddot{k}(n)}{\partial n}=-\ddot{k}(n) \frac{\alpha \sigma_{\eta}^{2} \tau n^{-2}}{1-\frac{\alpha}{n} \sigma_{\eta}^{2} \tau} .
$$

Since $\ddot{k}(n)$ is always positive, expression (2.8) is negative so long as the term $1-\frac{\alpha}{n} \sigma_{\eta}^{2} \tau$ is positive. This follows from assumption 1 and the assumption that $n$ is large enough to ensure $k^{*}$ interior.

What is the mechanism behind this result? Consider what happens as the authority increases revelation, starting from a very low level. As long as agents attend to all public signals, increasing revelation increases their learning about the state. At the threshold point $\widehat{n}$, however, agent $i$ no longer finds it worthwhile to attend to all signals, even if she believes that other agents do observe all signals. When this happens, the equilibrium cannot entail agents observing all signals. However, since each agent now observes only a subset of the public signals, each signal becomes less informative about others' actions, and therefore less valuable to agent $i$. Each agent is now less inclined to acquire even the previous quantity of signals, and aggregate information acquisition is reduced to a level below that obtained with slightly lower scope. 


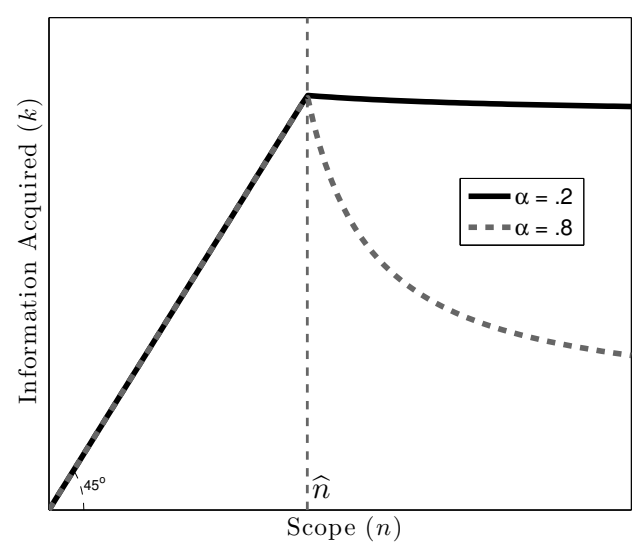

Figure 2: Information acquisition versus the scope of communication, for different degrees of strategic complementarity. Information acquisition is decreasing for scope greater than $\widehat{n}$.

One economic interpretation of this result is that over-communication on the part of the information authority, or central bank, results in harmful "cacophony" (Blinder, 2007). The central bank may, in principle, wish to communicate more information to the public, but speaking with too many voices (sending too many signals) may overload agents' interest or capacity to process that information. When this happens, extra communication is not only unhelpful, it actually reduces knowledge about the state in the private sector, which responds to the cacophony by collecting yet less information from the public announcements than it otherwise would.

Figure 2 plots the consequences of greater scope of communication for aggregate information acquisition. The figure shows that the effect of excessive scope on information acquisition can be substantial. When strategic complementarities are weak, information acquisition reaches a maximum at $\widehat{n}$, and declines only slowly thereafter. However, when complementarities are strong, the effect described above creates a dramatic reduction in information acquisition, to less than half of its 
maximum value as the number of signals released become arbitrarily large.

\subsection{Efficiency of Equilibrium Information}

Angeletos and Pavan (2007b) establish that $\alpha=\alpha^{\star}$ is sufficient to ensure that agents' equilibrium actions are efficient, taking their information as given. Proposition 3 extends this result to the endogenous choice of information, for a case with a unique equilibrium. This proposition explains the decision to call $\alpha=\alpha^{\star}$ the case of "preference alignment."

Proposition 3. Suppose $\alpha=\alpha^{\star}$. Then, given any communication policy, $\left\{n, \sigma_{\eta}^{2}\right\}$, the equilibrium of the model is a socially optimal information-action plan.

Proposition 3 is a consequence of a more general theorem proved in Chahrour (2011), which states under very general conditions on the cost of information that the socially optimal action plan is an equilibrium of the model. ${ }^{10}$ Other equilibria can (and often do) exist. For example, when assumption 1 does not hold, the model may have two equilibria, one in which agents acquire all of the authority's signals and one in which agents acquire no information. The different equilibria obviously have different welfare implications. The theorem establishes that one of the equilibria is efficient, but gives no guidance as to which that may be.

\footnotetext{
${ }^{10}$ Let the agent's information allocation consist of a reproduction alphabet, $\hat{M}$, and a conditional distribution $l(\hat{m} \mid \theta)$ on the message "received" by agent $i$, given the realization of the state. Then, for any information cost functional $c\left(\mathcal{I}^{i}\right)$ mapping information allocations to a real number, the optimal information-action plan is an equilibrium of the model.
} 


\section{Optimal Communication}

This section studies the consequences of endogenous information acquisition for the information authority's communication policy. I first establish the basic features of optimal scope and signal precision when agent's preference are aligned with the authority's. Next, I extend the model to study the case when the authority itself is uncertain about the realization of the state. Finally, I consider the choice of communication policy when agents and the authority value coordination to different degrees.

Definition 3 formally states the information authority's problem. The information authority selects its communication policy in order to maximize social welfare.

Definition 3. The information authority's optimal communication policy, denoted by $\left\{n^{\star}, \sigma_{\eta}^{2 \star}\right\}$, maximizes the social welfare of the resulting equilibrium allocations:

$$
\begin{aligned}
&\left\{n^{\star}, \sigma_{\eta}^{2^{\star}}\right\}=\underset{n, \sigma_{\eta}^{2}}{\operatorname{argmax}} U^{G}\left(k^{*}, p^{i^{*}}\left(\mathcal{I}^{i}\right) ; p(G)\right) \quad \text { subject to } \\
& p(G)=\int_{0}^{1} p^{i^{*}}\left(\mathcal{I}^{i}\right) d i ; \sigma_{\eta}^{2} \geq \underline{\sigma_{\eta}^{2}} ; \\
& k^{*}, p^{i^{*}}(\mathcal{I}) \text { are equilibrium allocations given policy } n .
\end{aligned}
$$

\subsection{Optimal Communication with Aligned Preferences}

In this section, I maintain the assumption that $\alpha=\alpha^{\star}$, and study the implications for optimal communication. I begin by focusing on this case for two reasons. First, it corresponds to the benchmark environment of firm price-setting decisions with imperfect competition (again see Hellwig, 2005; Roca, 2010). More generally, 
this case is relevant to communication policy in a wide range of economic environments. Second, this choice ensures that agents are using their information in an efficient manner, allowing me to isolate the consequences of the coordination problem created by excessive scope. In section 3.3, I examine the robustness of these results when $\alpha \neq \alpha^{\star}$.

\subsubsection{No-Waste Result}

Lemma 1 establishes that, under optimal communication policy, agents must attend to all signals released by the central bank.

Lemma 1. The optimal choice of scope induces agents to select $k=n$.

The intuitive proof (detailed in appendix C.1) is as follows. Pick any communication policy $n$ to the right of $\hat{n}$. The non-monotonicity of information acquisition implies the existence of policy $n^{\prime}$ to the left of $\hat{n}$, which achieves the same degree of information acquisition but ensures agents acquire all signals released by the authority. Consider the consequences of these two policies for social welfare. Under both policies, the information costs are equal and agents achieve the same precision of inference about the state. However, under policy $n^{\prime}$, agents' information is more correlated and their actions are more coordinated. Therefore, policy $n^{\prime}$ always strictly dominates the higher scope policy, $n$.

\subsubsection{Optimal Scope}

Using lemma 1, I can now compute the authority's preferred scope of communication. 
Proposition 4. The optimal choice of scope is given by

$$
n^{\star}=\sqrt{\frac{\sigma_{\eta}^{2}(1-\alpha)}{\lambda}}-(1-\alpha) \sigma_{\eta}^{2} \tau .
$$

Proposition 4 is established by 1) assuming full information acquisition on the part of agents, 2) maximizing the resulting social welfare function, and 3) checking that full information acquisition is indeed an equilibrium for the implied scope. The details of the proof are in appendix C.2.

Given the result in lemma 1, it might be tempting to guess that the authority seeks to maximize agents' information acquisition. Expression (3.2) immediately shows that this is not the case.

Corollary 2. When $\alpha=\alpha^{\star}>0$, the optimal scope is positive but entails providing fewer signals than agents would willingly acquire in equilibrium. That is,

$$
0<n^{\star}<\widehat{n}
$$

Figure 3 show this result graphically. With strong complementarities, the wedge between the number of signals agents would willingly acquire and the amount optimally provided by the authority is large. This result follows from the fact that higher complementarities decrease the social (and individual) value of learning about the state. To see this, consider the social welfare function when agents purchase all public information:

$$
-U^{G}\left(k^{*}, p^{i^{*}}(\mathcal{I}) ; p(G)\right)=(1-\alpha)\left(\left(n \psi_{1}+\psi_{2}-1\right)^{2}+\psi_{1}^{2} n \sigma_{\eta}^{2}\right)+\psi_{2}^{2} \sigma_{\xi}^{2}+\lambda n .
$$

When $\alpha$ is close to one, additional signals from the authority provide relatively 


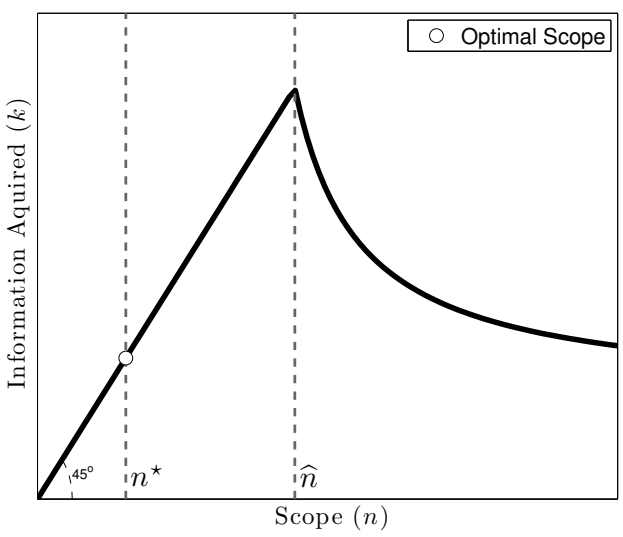

Figure 3: Information acquisition versus the scope of communication, for the case of strong complementarities $(\alpha=.8)$. The optimal degree of scope, $n^{\star}$, does not exhaust agents' willingness to acquire information.

little benefit because the "fundamental" portion of the loss function becomes small while the cost of each signal remains constant. As a result, the value of providing the public with more information is lower, reducing optimal scope.

Figure 4 provides additional intuition for this result. The figure plots the information cost paid by agent $i$ as a function of the correlation of her expectation of the state, conditional on the signals that she observes, with "market expectations" that are assumed to rely on the full set of signals released by the information authority. As the figure shows, higher levels of revelation increase the cost of informational coordination for agents. Since agents value coordination, they choose to pay this cost and acquire additional signals even beyond $n^{\star}$. Because of randomization, agent $i$ knows that any additional signals released beyond $n^{\star}$ will be observed by some others, and therefore will choose to acquire that signal. Since agent $i$ observes the signal, however, other agents also find it worthwhile. And so on. In order to ensure coordination, agents choose to purchase all the signals that are released, even though they would prefer that the signals were withheld entirely 


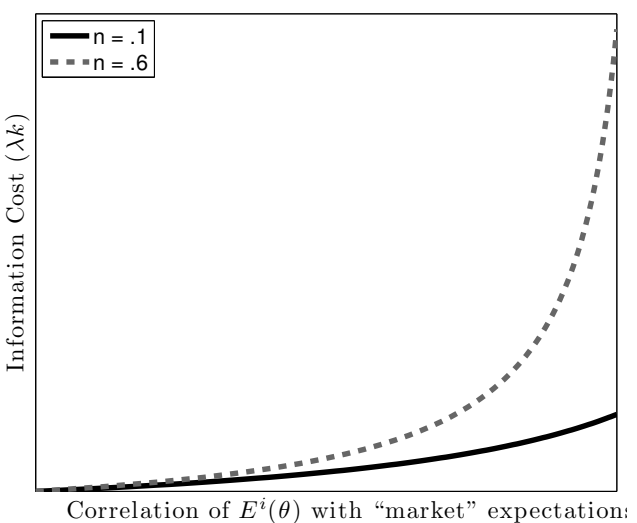

Figure 4: Information costs versus the degree of correlation with market information, for different degrees of scope. Higher scope increases the cost of coordinating information.

by the authority.

Since complementarities drive the desire to limit communication, a natural hypothesis is that the optimal scope of communication is increasing $\alpha$. In fact, this is only true when the exogenous private information is sufficiently imprecise.

Corollary 3. Optimal scope is decreasing in $\alpha$ if and only if

$$
\frac{\sigma_{\xi}^{2}}{2} \geq \sqrt{(1-\alpha) \lambda \sigma_{\eta}^{2}}
$$

The logic behind the result is that increasing the degree of complementarity both decreases the importance agents place on choosing actions that are close to the state and decreases the informativeness of private information for the optimal action. When private information is of low quality, agents rely relatively little on it and the latter effect is small: agents can coordinate sufficiently well with less public information, and so the authority provides fewer signals. On the other hand, when private information is relatively precise, an increase in complementarities causes the value of private information to fall more greatly, and the authority compensates 
for that loss by increasing information along the dimension it controls, namely increasing public signals.

These results bear a prima facie resemblance to those of Morris and Shin (2002): too much communication may be harmful and this harm is driven by complementarities in agents' actions. My results differ in crucial ways, however. First, the conclusions here are not the result of any sort of "misalignment" in preferences, the driving force behind the Morris and Shin (2002) result. Instead, they stem from the nature of communication in the model, namely that communication policy can be fashioned so as to facilitate coordination among agents, and that this is desirable. The economic intuition here is quite distinct from previous literature: in this paper, limiting the extent of communication serves to facilitate desirable coordination in agents' information, while in the Morris and Shin (2002) strand of literature it serves to temper over-coordination in agents' actions.

A second important difference is that the results here rationalize partial revelation, in which the authority communicates in a manner that is neither silent nor totally revealing. In contrast, the Morris and Shin (2002) result is "bang-bang" in nature: the authority should either release its message at the highest possible precision or not at all. In this model, if the authority were constrained to choose between full revelation or complete silence, it would indeed prefer full revelation. This last statement is a consequence of proposition 3, which states that when $\alpha=\alpha^{\star}$ equilibrium allocations are efficient for any given communication policy. Since zero public information is within each agent's choice set for any level of $n$, that they choose $k^{*}$ strictly greater than zero implies that positive scope dominates complete silence (in which case $k$ is constrained to be zero.) 


\subsubsection{Optimal Precision}

Proposition 5 establishes that, when preferences are aligned, the information authority always prefers to communicate as precisely as possible regardless of its choice of scope.

Proposition 5. Given n, social welfare is improving with communication quality. That is

$$
\frac{\partial U^{G}}{\partial \sigma_{\eta}^{2}}<0 .
$$

Proof of proposition 5. By proposition 3, it is sufficient to show that welfare is decreasing in $\sigma_{\eta}^{2}$, given a fixed level of $k$ : since agents' information choice given $\sigma_{\eta}^{2}$ is socially efficient, any response in information acquisition can only further increase welfare. But Angeletos and Pavan (2007a) prove that, when preferences are aligned, actions given information are efficient and a decrease in precision must be socially harmful. The result follows.

Since the precision result of Morris and Shin (2002) relies on a misalignment of preferences, proposition 5 may not be especially surprising. Yet, the model with endogenous information acquisition still calls for a limited scope of communication. This contrast highlights the distinction between the two dimensions of communication policy in the model. Even when the central bank wishes to limit the quantity of information it provides, it still would like its communications to be a precise as possible: there is no such thing as constructive ambiguity under the baseline preferences. In section 3.3, I extend this result to show that increases in the precision of public signals are welfare improving regardless of preference alignment, so long 
as the scope of communication is chosen optimally.

\subsection{Constraints on the Authority's own Information}

One question that arises frequently in the literature on transparency is whether the degree of the authority's knowledge about the state should impact how it communicates its message. The canonical model with a single public signal does not distinguish between limitations on the authority's knowledge of the state and limitations on its ability to clearly communicate that knowledge. In this section, I extend the model to allow for error in the authority's own knowledge of the state. The key characteristic of this type of error is that it is common across the authority's signals: every time it "speaks," the authority makes the same mistake because it misapprehends the realization of the state.

To this end, assume the authority learns about the state from a signal of the form $g=\theta+\varepsilon$. The authority, in turn, may freely release as many signals $g_{l}=$ $\theta+\varepsilon+\eta_{l}, l=1, \ldots, n$, as it wishes. The error term $\varepsilon$ is assumed to be independent of all other shocks and normally distributed with variance $\sigma_{\varepsilon}^{2}$. All other assumptions in the model are unchanged.

Let $\Gamma \equiv 1+\sigma_{\varepsilon}^{2}+(1-\alpha) \frac{\sigma_{\varepsilon}^{2}}{\sigma_{\xi}^{2}}$. Proposition 3.2 describes the equilibrium information choice of agents

Proposition 2(a). Suppose that assumption 1 holds and that $\sigma_{\varepsilon}^{2}$ is finite. Then the equilibrium information allocation is unique and is given by

$$
k^{*}= \begin{cases}n & \text { if } n \leq\left(\sqrt{\frac{\sigma_{\eta}^{2}}{\lambda}}-(1-\alpha) \sigma_{\eta}^{2} \tau\right) \frac{1}{\Gamma} \\ \ddot{k}(n) & \text { otherwise }\end{cases}
$$


where

$$
\ddot{k}(n)=\frac{\sqrt{\frac{\sigma_{\eta}^{2}}{\lambda}}-\sigma_{\eta}^{2} \tau}{\Gamma-\frac{\alpha}{n} \sigma_{\eta}^{2} \tau} .
$$

Since $\Gamma>1$, the threshold at which agents cease to observe all signals released by the authority necessarily shrinks. Furthermore, to the right of the threshold, the derivative

$$
\frac{\partial \ddot{k}(n)}{\partial \sigma_{\varepsilon}^{2}}=-\ddot{k}(n) \frac{\sigma_{\varepsilon}^{2}\left(1+\frac{1-\alpha}{\sigma_{\xi}^{2}}\right)}{\Gamma-\frac{\alpha}{n} \sigma_{\eta}^{2} \tau}<0 .
$$

Thus, agent's acquisition of public signals is always less when the authority knows less about the state.

The consequences for optimal scope of limits to the authority's own knowledge are considered in proposition 4(a).

Proposition 4(a). Suppose that assumption 1 holds and that $\sigma_{\varepsilon}^{2}$ is finite. Then the optimal degree of scope is given by

$$
n^{\star}=\left(\sqrt{\frac{\sigma_{\eta}^{2}(1-\alpha)}{\lambda}}-(1-\alpha) \sigma_{\eta}^{2} \tau\right) \frac{1}{\Gamma} .
$$

The proposition shows that optimal scope with $\sigma_{\varepsilon}^{2}>0$ is just a rescaling of the optimal level when the authority's information is perfect. Proposition 4(a) confirms an intuitive result: an authority which knows less about the state should provide fewer public signals about it.

Since errors in the authority's knowledge of the state are common across all public signals, they bear on the informativeness of the public signals with regard to the fundamental, but are not directly related to agents' coordination problem. 
For this reason, it is natural to suppose that increasing the magnitude of such errors is always harmful to social welfare. Proposition 6 establishes this result for the case of aligned preferences.

Proposition 6. Given n, equilibrium social welfare is increasing in the precision of the authority's own information. That is,

$$
\frac{\partial U^{G}}{\partial \sigma_{\varepsilon}^{2}}<0 .
$$

Proof. Again, by proposition 3, it is sufficient to show this holds for a given choice of $k$. The proposition then follows directly from the fact that actions given information are efficient when preferences are aligned.

In summary, the addition of uncertainty in the authority's own apprehension of the state confirms some natural conjectures, but it does not affect the basic mechanism of the model. The results on information acquisition and optimal scope follow with minimal modification.

\subsection{Optimal Communication with Misaligned Preferences}

So far, I have emphasized the case where agents and the social planner have identical preferences. In this case, the degree to which coordination is desirable (both socially and from the agents perspective) drives the choice of scope. Yet, Morris and Shin (2002) and the subsequent literature emphasize the link between preference misalignment (or externalities) and the choice of communication policy. In this section, I examine the interaction between the information acquisition 
mechanism I have detailed so far, and the inefficiencies (in actions and information acquisition) that may arise when agents and the authority have different preferences. Although the basic conclusions regarding optimal communication survive, substantial nuances emerge when agents are endowed with a very precise private signal. I therefore divide this section in two, focusing first on the extreme case of no private information, before turning to the model in its full generality.

\subsubsection{No Private Information}

In this section, I assume that agents can only access information about the aggregate via the pronouncements of the information authority. That is, I assume

that $\sigma_{\xi}^{2} \rightarrow \infty$. In this case, the no-waste result holds and the authority's desire for coordination completely drives the choice of scope.

Lemma 1(b). The optimal choice of scope induces agents to select $k=n$.

The logic behind lemma $1(\mathrm{~b})$ is exactly that of lemma 1, with one exception. When $k<n$, actions are generally inefficient. In this case, the authority has greater incentive to choose scope to ensure full acquisition. It is only in this case that equilibrium action coefficients are equal to the coefficients of inference, and the inefficiency in actions is eliminated.

Proposition 4(b). The optimal degree of scope is given by

$$
n^{\star}=\sqrt{\frac{\sigma_{\eta}^{2}\left(1-\alpha^{\star}\right)}{\lambda}}-\sigma_{\eta}^{2} .
$$

Proof of Proposition 4(b). Because there is no inefficiency in actions so long as $k \leq \widehat{n}$, agents' own complementarities have no effect on social welfare. Furthermore, 


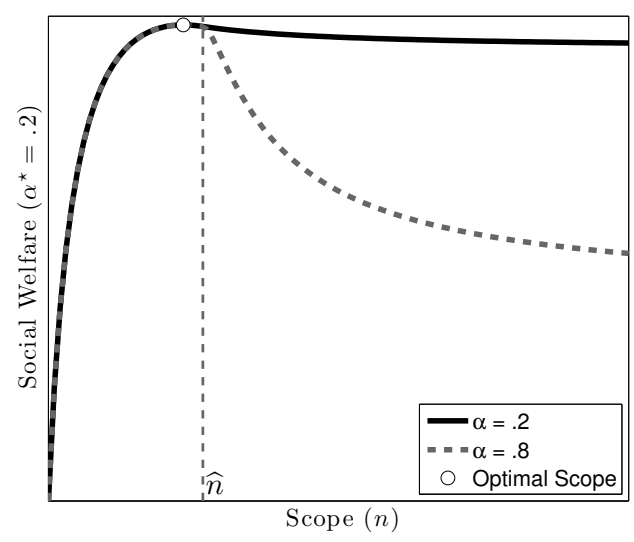

Figure 5: Social welfare as a function of the scope of communication, when agent's and the planner's preferences are misaligned. When $\alpha^{\star}=.2$, high complementarities perceived by agents imply large losses to scope greater than $\widehat{n}$.

when there is no private information, the threshold level $\widehat{n}=\sqrt{\frac{\sigma_{\eta}^{2}}{\lambda}}-\sigma_{\eta}^{2}$ does not depend on $\alpha$. In this case, the authority can achieve its preferred outcome irrespective of $\alpha$, and this outcome is achieved by $n^{\star}$.

Despite the strong result in proposition 4(b), private complementarity has important implications for the consequences of sub-optimal levels of scope. Figure 5 , compares social welfare for different degree of private complementarities, when $\alpha^{\star}=.2$. When complementarities are low, "excessive" scope has very small consequences for social welfare, since information acquisition is little affected by the extra revelation. However, when complementarities are strong, agents react to additional signals by dramatically reducing their own acquisition of information. In this case, social welfare may be greatly harmed by a relatively small amount of excess scope. Importantly, however, this wedge only appears once scope exceeds the level $\widehat{n}$ at which agents cease to purchase all public information. As a result, the impact of small errors in the degree of scope depends only on $\alpha^{\star}$; $\alpha$ is only relevant in the range where information acquisition decreases with scope. 
Another natural question is whether preference mis-alignment could reverse the conclusion that the authority prefers to provide the most precise signal possible. Proposition 5(b) establishes that, without private information, this result does not change.

Proposition 5(b). The optimal communication policy entails signals of maximum precision:

$$
\sigma_{\eta}^{2^{*}}=\underline{\sigma_{\eta}^{2}}
$$

Proof of Proposition 5(b). Since optimal scope must ensure full information acquisition, the result is immediate. Actions are optimal as long as information is common across all agents and, when actions are socially efficient, increases in the signal precision increases social welfare.

Unlike proposition 5, proposition 5(b) only applies to the joint choice of precision and scope. Yet, the proof establishes that welfare is increasing in precision for any fixed level of scope that induces full acquisition. For a fixed level of scope beyond this threshold, however, greater precision of communication may indeed harm welfare. Surprisingly, however, this may only be true when $\alpha<\alpha^{\star}$. Why? This result follows from the exclusion of purely private information. In this case, when agents observe a fraction of the authority's signals, their observations become become relatively private, compared to the common prior held by all agents. Following Hellwig (2005), agents tend to overweight such signals when $\alpha<\alpha^{\star}$. Because they overvalue private information, agents will also tend to over-acquire these signals. In some instances with high scope, these inefficiencies can swamp the benefit of an increase in the precision of each signal. The authority, however, avoids this outcome by optimally ensuring that all agents receive the same message. 


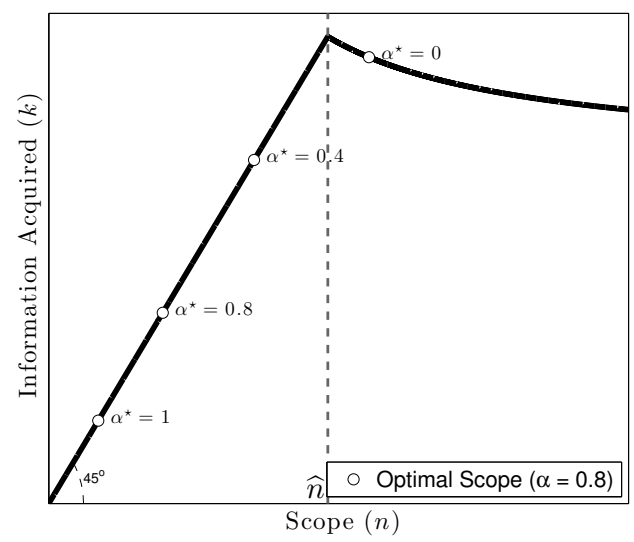

Figure 6: Optimal scope when preferences are misaligned. When preference misalignment is strong $\left(\alpha=0.8, \alpha^{\star}=0\right)$, optimal scope may exceed the threshold level, $\widehat{n}$.

\subsubsection{Private Information}

Once endowed with a private signal, agents' actions may be inefficient even when they observe every signal released by the authority. This introduces a new "policy target" for the authority: it must now use its communications to influence both equilibrium information choice and the equilibrium use of that information. In some circumstances the authority may choose scope beyond the threshold $\widehat{n}$. In these cases, the authority uses agent's inability to coordinate information to its advantage: by releasing more signals it ensures that agents experience some dispersion in their information, and therefore respond in a more muted way to each signal.

Proposition 4(c). The optimal level of scope is finite:

$$
n^{\star}<\infty
$$

To build some intuition for this general result, figure 6 shows optimal scope 
for different values $\alpha^{\star}$. When $\alpha \leq \alpha^{\star}$, a no-waste theorem applies and finite optimal scope follows immediately. On the other hand, when $\alpha>\alpha^{\star}$, it is indeed possible that the authority benefits from inducing some dispersion among agent's information by increasing scope beyond $\widehat{n}$. In this case, the finiteness of optimal scope follows from the presence of a common prior and the fact that the planners' preferred level of information acquisition lies above the equilibrium level. Increases in scope decrease both the level of information acquisition and the correlation of agent's signals. When agents share a prior, however, purely private information is underweighted by agents in their actions: conditional on the level of information acquisition, the authority does not desire for agents to receive purely idiosyncratic messages, as would occur with unbounded scope. Nor does the authority desire to reduce information acquisition below already inefficiently low levels. Thus, the planner necessarily settles on a finite level of scope. See appendix E.1 for the details of this proof.

Proposition 5(c). The optimal communication policy entails signals of maximum precision:

$$
\sigma_{\eta}^{2^{\star}}=\underline{\sigma_{\eta}^{2}}
$$

The argument (detailed in the appendix) is essentially as follows. Consider a small increase of precision from $\sigma_{\eta}^{2}$ to $\sigma_{\eta}^{2 \prime}$. For the first case, assume the authority had optimally selected scope to ensure full acquisition when the precision of its signals was $\sigma_{\eta}^{2}$. Then it can always achieve higher welfare by reducing the number of signals to achieve the same precision on the combined public signal while decreasing agents' expenditure on information. For the second case, assume the authority had selected a level of scope at which agents do not acquire all signals. Taking into 
account agent information choice, it is once again always possible for the authority to change scope so as to ensure that agents maintain the precision of their inference. In doing so, however, both the information costs paid and the correlation of agents' information are reduced, leading to a benefit for the planner.

The result in proposition 5(c) bears some similarity to the conclusion of Cornand and Heinemann (2008) that, when public information is shared with only a fraction of the population and that fraction is selected optimally, higher precision of the public signal is always desirable. In their model, revealing a public message to a smaller fraction of the population represents an alternative, and less costly, means of inhibiting over-coordination in agents' actions. Here, revealing more information causes agents to endogenously receive more dispersed messages. In each case, the authority prefers to use an alternative dimension of communication policy, rather than reduce the precision of its message.

\section{Directed Search}

So far, I have assumed that agents cannot direct their search towards any particular signals among those released by the authority. Since this is a strong assumption, I now consider the consequences of relaxing it. In this section, I argue that such undirected search can be supported as an equilibrium outcome in a version of the model in which agents choose the probability with which they observe individual signals. Furthermore, when the cost of directing search is sufficiently high, this is the only equilibrium of the model. On the other hand, when directed search is free, the model admits a wide-range of equilibria with different welfare implications. For simplicity, in this section I focus on the special case in which 
preferences are aligned $\left(\alpha=\alpha^{\star}\right)$, agents have no exogenous private information $\left(\sigma_{\xi}^{2}=\infty\right)$, and the authority knows the state perfectly $\left(\sigma_{\varepsilon}^{2}=0\right)$.

Now, assume that in addition to choosing how many signals overall to observe, agent $i$ can also assign a sequence of weights $\mu_{1}, \mu_{2}, \ldots, \mu_{n}$, denoting the probability that signal $i$ is among those selected. The weights must be between zero and one and respect the restriction $\sum_{l=1}^{n} \mu_{l}=k .^{11}$ In order to make their observations, agents must pay a cost $c\left(\left\{\mu_{l}\right\}\right)$. I assume that the function $c$ is symmetric, continuously differentiable, and increasing in each of its arguments. Agent's information allocation now consists of the set $\left\{\mu_{l}\right\}$, however the definition of equilibrium in the extended model is otherwise identical to definition in the baseline case.

In appendix F, I once again extend the model to a version with continuous rather than discrete choices. The solution to agent $i$ 's problem is then characterized by

$$
\underset{\mu(l), k}{\operatorname{argmax}} U^{i} \quad \text { subject to } \quad \mu(l) \leq 1 ; \mu(l) \geq 0 ; \int_{0}^{n} \mu(l)=k .
$$

\subsection{A Condition for Uniqueness}

I now suppose a particular functional form for $c(\mu(l))$ and show that, under a plausible restriction on the cost of information, uniqueness is recovered in the model. In particular, I assume that $c(\mu(l))$ is given by the CES aggregator,

$$
c(\mu(l))=\lambda n^{\frac{\omega-1}{\omega}}\left(\int_{0}^{n} \mu(l)^{\omega}\right)^{\frac{1}{\omega}} .
$$

The parameter $\omega \geq 1$ measures the cost of directed search, in that $c(\mu(l))$ is increasing in the dispersion of $\mu(l)$ for a given $k$. That is, when $\omega>1$, agents pay

\footnotetext{
${ }^{11}$ Under the baseline assumption of undirected search, $\mu_{l}=\mu_{j}=\frac{k}{n}$ and $\sum_{l=1}^{n} \mu_{l}=n \frac{k}{n}=k$.
} 
more to sample the same number of signals, to the extent that they act to observe certain signals of the authority and not others. The $\frac{\omega-1}{\omega}$ exponent of $n$ is selected to ensure that, when $\mu(l)$ is constant for all $l$, this specification nests the linear cost function used earlier in the paper.

Assumption 2. The costs of coordinating information are high:

$$
\omega>\frac{1+\alpha}{1-\alpha}
$$

Proposition 7 establishes that, for a sufficiently high cost of search, the equilibrium of the model is exactly that studied under the baseline assumptions.

Proposition 7. Suppose that the cost of information is given by the CES aggregator in equation (4.2) and $\omega$ satisfies assumption 2. Then equilibrium of the model is unique, and is characterized by

$$
\mu(l)=\frac{k^{*}}{n}, \forall l
$$

In this case, the policy and welfare implications are identical to the baseline model.

This condition is sufficient for equilibrium uniqueness, but not necessary. For $\omega \in\left(1, \frac{1-\alpha}{1+\alpha}\right)$, the presence and extent of multiplicity will depend on the other parameters of the model. However, for these cases, the complete set of equilibria is difficult to characterize in closed form. 


\subsection{Efficiency of the Directed Search Equilibria}

In contrast to above uniqueness result, Hellwig and Veldkamp (2009) emphasize the potential for multiplicity in environments with endogenous information acquisition. Indeed, when the costs of coordinating information do not satisfy assumption 2, the model may have (and indeed typically does have) multiple equilibria. The different equilibria entail different levels of social welfare. However, whenever $\alpha=\alpha^{\star}$, the socially optimal information-action plan satisfying the efficiency criterion in definition 2 is always among the equilibria. ${ }^{12}$ This fact follows from the previously cited theorem in Chahrour (2011).

\subsection{Robust Communication Policy}

In this section, I study a version of the model in which the equilibrium information choice is not unique and study the consequences for scope. The simplest case is that in which the cost of information is linear in the number of signals sampled. This corresponds to the limiting CES case where $\omega=1$, so that cost of information is $c(\mu(l))=\lambda \int_{0}^{n} \mu(l) d l=\lambda k$. The set of solutions to the first order conditions (described in proposition 9 in appendix F.4) is no longer unique and includes cases where agents direct their search perfectly for all signals (choose $\mu(l) \in\{0,1\}$ for all l), direct their search perfectly for some signals but randomly sample over others, and sample all signals with equal probability. ${ }^{13}$

The different equilibria have different welfare implications. If the central bank were able to choose its preferred equilibrium from among the set of all equilibria,

\footnotetext{
${ }^{12}$ It is important to recall that this statement concerns efficiency given a particular communication policy.

${ }^{13}$ Again this multiplicity is over and above the trivial sort of multiplicity that arises from interchanging the identities of the a priori identical signals.
} 


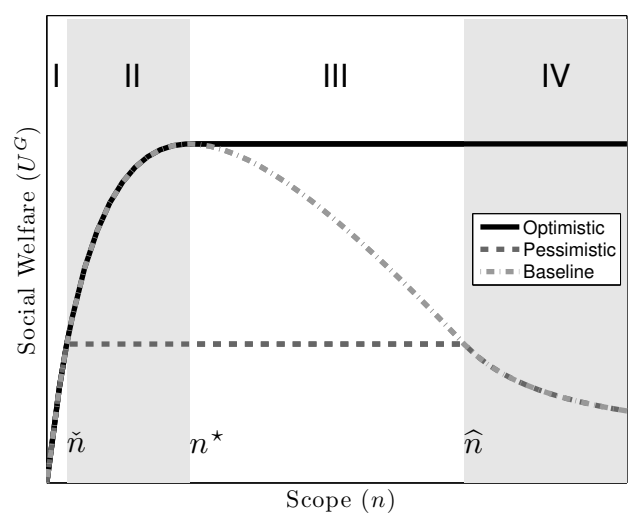

Figure 7: Social welfare for alternative equilibrium scenarios. Robust (min-max) communication policy selects $n^{\star}$ within region III.

then its choice of scope beyond $n^{\star}$ would be irrelevant, since it could always direct agents to ignore (its own!) extraneous signals. Such an authority could be said to be "optimistic" about the potential equilibrium outcome given its policy choice. However, if the authority is concerned that another, less desirable, equilibrium might emerge, then it may wish to take the full set of possible equilibria into account. A simple way to do this is to consider worst case scenarios - the information equilibrium with the lowest welfare - for differing degrees of scope. Such a "pessimistic" authority could then choose scope in order to place a lower-bound on the equilibrium outcome.

Figure 7 compares welfare for the best and worst case scenarios, along with the welfare generated under the baseline assumption that an agent must sample all signals with equal probability. Note that this last outcome (i.e. that of randomizing among all signals) is always an equilibrium of the model with free directed search. I now divide the range of scope into four regions, and describe the characteristics of each.

For extremely low levels of scope denoted by region I, all three welfare measures 
coincide. In these cases, the unique equilibrium is one in which agents acquire all of the signals released by the information authority. In this region, agents find it worthwhile to acquire a marginal signal, regardless of whether other agents acquire it also.

In region II, the optimistic authority anticipates that agents will acquire all signals, while the pessimistic authority fears one of the (equally) bad outcomes in which information agents either acquire too-little information, engage in undirected search across signals, or both. Agents are therefore too-little informed about the realization of the state or too-little coordinated in their information. Once scope surpasses $\check{n}$, worst case welfare is invariant to scope, but the set of equilibria yielding this outcome becomes larger. Meanwhile, for the baseline version of the model, the randomization assumption ensures that agents acquire all information. This is desirable throughout region II, and so the baseline model corresponds with the "best equilibrium" in this region.

In region III, the optimistic central bank remains assured that agents will focus their search on only the number of signals it knows is optimal, ignoring all others. Again, the pessimistic authority fears that agents will focus their attention too narrowly, ignoring worthwhile information, or engage in undirected search. Under the baseline assumption of sampling all signals equally, however, the agents continue to acquire all information. This outcome is suboptimal relative to the social action plan, but still better than the low-information equilibrium. Finally, in region IV, the worst-case scenario coincides with the equal-probability search assumption, as coordination decreases even as agents acquire too much information from a social perspective.

The figure suggests that a robust communication policy should place the au- 
thority somewhere in region II or region III. In these regions, the worst case scenario entails the smallest social loss. Moreover, choosing scope in region III ensures that the best case scenario remains among the set of equilibria. Finally, note that the best case, which corresponds to the social planner's optimal information-action plan, is also the outcome under the baseline model at $n^{\star}$. If the authority communicates with the optimal scope, then it has no incentive to facilitate directed search on the part of agents. Instead, it benefits if agents believe that any signal it releases is observed by others, as this ensures that each agent has an individual incentive to acquire it.

\section{Conclusions and Extensions}

This paper offers a new explanation for why central banks often decline to provide as much information as possible about their own views on the economy. As in the previous literature on the social value of public information, my results depend on the presence of strategic complementarities: without these strategic incentives, limiting the scope or precision of public communication never improves social welfare. Yet, in contrast to this earlier literature, my results do not rely on any sort of misalignment in preferences from the agents' and social planner's perspective. Thus, the endogenous information model used here expands the variety of contexts in which incomplete information revelation by a public authority can be optimal.

Although my model rationalizes limits on the scope of communication, it also implies that public communications should always be as precise as possible. This result helps explain why even the most ardently "transparent" central banks strictly control their public communications. My results also explain why a central bank, 
or other authority, might resort to partial revelation rather than choosing either to provide as much information as possible, or to shut down communication entirely. Avoiding such "bang-bang" outcomes increases the plausibility of the results because it accords more closely with the observation that public agencies typically release substantial but limited amounts of information.

Finally, my paper highlights the point that agents' willingness to acquire a bit of public information need not imply that releasing this information is optimal. More communication increases the cost agents must pay to coordinate information, and increasing this cost is counterproductive if coordination is socially beneficial. Conversely, when agents tend to over-coordinate on public information, releasing additional signals may be beneficial precisely because all agents do not acquire them. By communicating more, the central bank can prevent agents from relying too much on a very small set of public communications.

Since communication is fundamentally about the transfer of information, the introduction of agents' information choice imposes new discipline on the study of optimal communication. One interesting direction for further research would be to study how costly information affects the ability of the authority to commit to truthful communication, as "cheap talk" is explicitly excluded from the current environment. Public communication is also widely viewed as an essential component to commitment in monetary policy itself (for example, see Woodford, 2005.) Modeling information choice and endogenous policy together would allow further investigation of this important interaction. 


\section{References}

Allen, Franklin, Stephen Morris, and Hyun Song Shin, "Beauty Contests and Iterated Expectations in Asset Markets," The Review of Financial Studies, 2006, 19 (3), pp. 719-752.

Amador, Manuel and Pierre-Olivier Weill, "Learning From Prices: Public Communication and Welfare," The Journal of Political Economy, 2010, 118 (5), pp. 866-907.

Angeletos, George-Marios and Alessandro Pavan, "Transparency of Information and Coordination in Economies With Investment Complementarities," The American Economic Review, 2004, 94 (2), pp. 91-98.

_ and _ , "Efficient Use of Information and Social Value of Information," Econometrica, 2007, 75 (4), pp. 1103-1142.

_ and _, "Socially Optimal Coordination: Characterization and Policy Implications," Journal of the European Economic Association, 2007, 5 (2-3), 585 593.

Blinder, Alan S., "Monetary Policy By Committee: Why and How?," European Journal of Political Economy, 2007, 23 (1), 106 - 123. Central bank transparency and central bank communication.

Chahrour, Ryan, "Central Bank Communication under Rational Inattention," 2011. Working Paper. 
Chen, Xiang-Hui, Arthur P. Dempster, and Jun S. Liu, "Weighted Finite Population Sampling to Maximize Entropy," Biometrika, 1994, 81 (3), pp. 457469.

Colombo, Luca and Gianluca Femminis, "The Social Value of Public Information With Costly Information Acquisition," Economics Letters, 2008, 100 (2), $196-199$.

_, _, and Alessandro Pavan, "Information Acquisition and Welfare," April 2012. Working Paper.

Cornand, Camille and Frank Heinemann, "Optimal Degree of Public Information Dissemination," Economic Journal, 2008, 118 (528), 718 - 742.

Dewan, Torun and David P. Myatt, "The Qualities of Leadership: Direction, Communication, and Obfuscation," American Political Science Review, 2008, $102(03), 351-368$.

Hellwig, Christian, "Heterogeneous Information and the Benefits of Transparency," 2005. Working Paper, UCLA.

- and Laura Veldkamp, "Knowing What Others Know: Coordination Motives in Information Acquisition," Review of Economic Studies, 2009, 76 (1), 223 251.

Judd, Kenneth L., "The Law of Large Numbers With a Continuum of IID Random Variables," Journal of Economic theory, 1985, 35 (1), 19-25. 
Kool, Clemens, Menno Middeldorp, and Stephanie Rosenkranz, "Central Bank Transparency and the Crowding Out of Private Information in Financial Markets," Journal of Money, Credit and Banking, 2011, 43 (4), 765-774.

Llosa, Luis Gonzalo and Venky Venkateswaran, "Efficiency With Endogenous Information Choice," March 2012. Working Paper.

Malenko, Nadya, "Communication and Decision Making in Corporate Boards," 2011. Working Paper.

McCall, John J., "Exchangeability and Its Economic Applications," Journal of Economic Dynamics and Control, 1991, 15 (3), 549 - 568.

Morris, Stephen and Hyun Song Shin, "Social Value of Public Information," The American Economic Review, 2002, 92 (5), pp. 1521-1534.

_ and _, "Optimal Communication," Journal of the European Economic Association, 2007, 5 (2-3), 594-602.

Myatt, David P. and Chris Wallace, "Endogenous Information Acquisition in Coordination Games," August 2010. Discussion Paper.

Reis, Ricardo, "Inattentive Producers," The Review of Economic Studies, 2006, 73 (3), pp. 793-821.

_, "When Should Policy Makers Make Announcements," March 2010. Working Paper.

Roca, Mauro, "Transparency and Monetary Policy With Imperfect Common Knowledge," 2010. IMF Working Paper. 
Sims, Christopher A., "Implications of Rational Inattention," Journal of Monetary Economics, 2003, 50 (3), 665 - 690. Swiss National Bank/Study Center Gerzensee Conference on Monetary Policy under Incomplete Information.

Svensson, Lars E. O., "Social Value of Public Information: Comment: Morris and Shin (2002) is Actually Pro-Transparency, Not Con," The American Economic Review, 2006, 96 (1), pp. 448-452.

Ueda, Kozo, "Central Bank Communication and Multiple Equilibria," International Journal of Central Banking, 2010, 6 (3), 145-167.

Uhlig, Harald, "A Law of Large Numbers For Large Economies," Economic Theory, 1996, 8 (1), $41-50$.

Vives, Xavier, "Endogenous Public Information and Welfare," 2010. Working Paper.

Wong, Jacob, "Information Acquisition, Dissemination, and Transparency of Monetary Policy," Canadian Journal of Economics, 2008, 41 (1), 46 - 79.

Woodford, Michael, "Central Bank Communication and Policy Effectiveness," Working Paper 11898, National Bureau of Economic Research December 2005. 


\section{A Computing Equilibrium}

\section{A.1 Inference}

Agent $i$ 's observations can be collected in the vector $y=\left[g_{1}, g_{2}, \ldots, g_{k}, r^{i}\right]$, which can be rewritten as $y=H^{\prime} x$ where $x=\left[\theta, \eta_{1}, \ldots, \eta_{k}, \xi^{i}\right]$ and $Q=\operatorname{cov}(x)$. Under the normality assumption, optimal signal extraction implies that

$$
E(x \mid y)=(Q H)\left(H^{\prime} Q H\right)^{-1} y
$$

The coefficient $\gamma_{1}$ comprises the first $n$ entries of the first row of the matrix $(Q H)\left(H^{\prime} Q H\right)^{-1}$ and $\gamma_{2}$, the last entry of that row.

\section{A.2 Equilibrium Actions}

In this section, I solve for the equilibrium coefficients of the agent's action rule, taking the information structure $k \leq n$ as given. I conjecture that the aggregate action rule takes the form given in equation (2.1), derive agent $i$ 's optimal response, and compute aggregate actions given the hypothesized rule. Equilibrium is a fixed point of the resulting mapping.

Throughout, I denote with a tilde any equilibrium objects taken as given by agent $i$. For example, since agent $i$ takes aggregate actions as given, the aggregate action from the perspective of agent $i$ is assumed to be $\mathrm{e}^{14}$

$$
p=\widetilde{\psi_{1}} \sum_{l=1}^{n} g_{l}+\widetilde{\psi_{2}} \theta .
$$

\footnotetext{
${ }^{14}$ Myatt and Wallace (2010) discuss the mild restrictions required to ensure the equilibrium takes a linear form.
} 
Let $\gamma_{1}$ and $\gamma_{2}$ be defined as in the text. Then, agent $i$ 's expectation of the state and aggregate price are given respectively by

$$
\begin{aligned}
& E\left(\theta \mid \mathcal{I}^{i}\right)=\gamma_{1} \sum_{l=1}^{n} \mathbb{1}\left[g_{l} \in \mathcal{I}^{i}\right] g_{l}+\gamma_{2} r^{i} \\
& E\left(p \mid \mathcal{I}^{i}\right)=\widetilde{\psi_{1}} \sum_{l=1}^{n} E\left(g_{l} \mid \mathcal{I}^{i}\right)+\widetilde{\psi_{2}} E\left(\theta \mid \mathcal{I}^{i}\right) .
\end{aligned}
$$

Since I assume that agents know the identity of the signals they have observed, the conditional expectation of signal $l$ is given by

$$
E\left(g_{l} \mid \mathcal{I}^{i}\right)= \begin{cases}g_{l} & \text { if } g_{l} \in \mathcal{I}^{i} \\ E\left(\theta \mid \mathcal{I}^{i}\right) & \text { if } g_{l} \notin \mathcal{I}^{i} .\end{cases}
$$

After some simplification, we can compute the expectation of the aggregate action

$$
E\left(p \mid \mathcal{I}^{i}\right)=\left(\widetilde{\psi_{1}}\left(1+(n-k) \gamma_{1}\right)+\widetilde{\psi_{2}} \gamma_{1}\right) \sum_{l=1}^{n} \mathbb{1}\left[g_{l} \in \mathcal{I}^{i}\right] g_{l}+\gamma_{2}\left(\widetilde{\psi_{1}}(n-k)+\widetilde{\psi_{2}}\right) r^{i}
$$

Evaluating the agent first order condition in expression (1.2), we get agent $i$ 's choice of action as a function of her observations:

$$
\begin{aligned}
p^{i}= & \left((1-\alpha) \gamma_{1}+\alpha\left(\widetilde{\psi_{1}}\left(1+(n-k) \gamma_{1}\right)+\widetilde{\psi_{2}} \gamma_{1}\right)\right) \sum_{l=1}^{n} \mathbb{1}\left[g_{l} \in \mathcal{I}^{i}\right] g_{l} \\
& +\gamma_{2}\left(1-\alpha+\alpha\left(\widetilde{\psi_{1}}(n-k)+\widetilde{\psi_{2}}\right)\right) r^{i} .
\end{aligned}
$$


Rearranging the weights on the public and private signals in equation (A.7), define

$$
\begin{aligned}
& \psi_{1}^{i} \equiv \alpha \widetilde{\psi_{1}}+\gamma_{1}\left(1-\alpha+\alpha\left(\widetilde{\psi_{1}}(n-k)+\widetilde{\psi_{2}}\right)\right) \\
& \psi_{2}^{i} \equiv \gamma_{2}\left(1-\alpha+\alpha\left(\widetilde{\psi_{1}}(n-k)+\widetilde{\psi_{2}}\right)\right)
\end{aligned}
$$

to be the coefficients of agent $i$ 's optimal action rule, given (any) aggregate coefficients $\widetilde{\psi_{1}}$ and $\widetilde{\psi_{2}}$. In order to compute the average action, I must compute the cross-sectional average of $\mathbb{1}\left[g_{l} \in \mathcal{I}^{i}\right] g_{l}{ }^{15}$ By assumption, the set of signals observed is unrelated to the realizations of the signals themselves. Thus, this is just $E\left(\mathbb{1}\left[g_{l} \in \mathcal{I}^{i}\right]\right) g_{l}=\operatorname{prob}\left(g_{l} \in \mathcal{I}^{i}\right) g_{l}$. Because sampling is purely random, all possible combinations of signals observed are equally likely and we can immediately conclude that $\operatorname{prob}\left(g_{l} \in \mathcal{I}^{i}\right)=\frac{k}{n}$.

Using this fact, I integrate equation (A.7) across agents to arrive at an expression for the aggregate action:

$$
\begin{aligned}
p= & \frac{k}{n}\left((1-\alpha) \gamma_{1}+\alpha\left(\widetilde{\psi_{1}}\left(1+(n-k) \gamma_{1}\right)+\widetilde{\psi_{2}} \gamma_{1}\right)\right) \sum_{l=1}^{n} g_{l} \\
& +\gamma_{2}\left((1-\alpha)+\alpha\left(\widetilde{\psi_{1}}(n-k)+\widetilde{\psi_{2}}\right)\right) \theta
\end{aligned}
$$

Comparing equations (A.2) and (A.10), I conclude that the equilibrium coefficient is a fixed point of the recursive relationship

$$
\left[\begin{array}{c}
\psi_{1}^{\prime} \\
\psi_{2}^{\prime}
\end{array}\right]=(1-\alpha)\left[\begin{array}{c}
\frac{k}{n} \gamma_{1} \\
\gamma_{2}
\end{array}\right]+\alpha\left[\begin{array}{cc}
\frac{k}{n}\left(1+(n-k) \gamma_{1}\right) & \frac{k}{n} \gamma_{1} \\
(n-k) \gamma_{2} & \gamma_{2}
\end{array}\right]\left[\begin{array}{l}
\psi_{1} \\
\psi_{2}
\end{array}\right]
$$

\footnotetext{
${ }^{15}$ See Judd (1985); Uhlig (1996) for a discussion of the issues related to using a law of large numbers when integrating across a continuum of agents.
} 
Solving for the fixed point and substituting in for $\gamma_{1}$ and $\gamma_{2}$ yields the expressions

$$
\begin{aligned}
& \psi_{1}^{*}=\left(n+\left(\frac{n}{k}-\alpha\right) \sigma_{\eta}^{2}\left(\frac{1}{1-\alpha}+\frac{1}{\sigma_{\xi}^{2}}\right)\right)^{-1} \\
& \psi_{2}^{*}=\left(1+\sigma_{\xi}^{2}\left(\frac{1}{1-\alpha}+\frac{k}{1-\alpha \frac{k}{n}} \frac{1}{\sigma_{\eta}^{2}}\right)\right)^{-1} .
\end{aligned}
$$

\section{A.3 Morris and Shin (2002) Effect}

Under the assumption that $n=k=1$ and $\alpha^{\star}=0$, the equilibrium action coefficients are given by

$$
\begin{aligned}
& \psi_{1}^{*}=\left(1+\sigma_{\eta}^{2}\left(1+\frac{1-\alpha}{\sigma_{\xi}^{2}}\right)\right)^{-1} \\
& \psi_{2}^{*}=\left(1+\frac{\sigma_{\xi}^{2}}{1-\alpha}\left(1+\frac{1}{\sigma_{\eta}^{2}}\right)\right)^{-1} .
\end{aligned}
$$

Social losses are given by

$$
-U^{G}=\left(\psi_{1}^{*}+\psi_{2}^{*}-1\right)^{2}+\left(\psi_{1}^{*}\right)^{2} \sigma_{\eta}^{2}+\left(\psi_{2}^{*}\right)^{2} \sigma_{\xi}^{2} .
$$

Taking the derivative with respect to $\sigma_{\eta}^{2}$ yields

$$
\frac{\partial U^{G}}{\partial \sigma_{\eta}^{2}}=-\frac{\left(\sigma_{\xi}^{2}\right)^{2}\left(\sigma_{\xi}^{2}\left(1+\sigma_{\eta}^{2}\right)+(1-2 \alpha)(1-\alpha) \sigma_{\eta}^{2}\right)}{\left(\sigma_{\xi}^{2}\left(1+\sigma_{\eta}^{2}\right)+(1-\alpha) \sigma_{\eta}^{2}\right)^{3}}
$$

which is greater than zero if any only if

$$
(2 \alpha-1)(1-\alpha)>\sigma_{\xi}^{2}\left(1+\frac{1}{\sigma_{\eta}^{2}}\right) .
$$




\section{A.4 Equilibrium Information}

I now solve for agent $i$ 's choice of information, taking as given aggregate information and the equilibrium mapping of information to actions. Following the derivation above, the aggregate price from the perspective of agent $i$ is given by the linear rule (A.2), where

$$
\begin{aligned}
& \widetilde{\psi_{1}}=\left(n+\left(\frac{n}{\widetilde{k}}-\alpha\right) \sigma_{\eta}^{2}\left(\frac{1}{1-\alpha}+\frac{1}{\sigma_{\xi}^{2}}\right)\right)^{-1} \\
& \widetilde{\psi_{2}}=\left(1+\sigma_{\xi}^{2}\left(\frac{1}{1-\alpha}+\frac{\widetilde{k}}{1-\alpha \frac{\widetilde{k}}{n}} \frac{1}{\sigma_{\eta}^{2}}\right)\right)^{-1} .
\end{aligned}
$$

Suppose that agent $i$ selects to observe $k$ signals and reacts to her information optimally according to the first order condition given by (1.2). Using the weights from agent $i$ 's action rule in (A.8)-(A.9), we can compute the differences

$$
\begin{aligned}
p^{i}-\theta= & \left(k \psi_{1}^{i}+\psi_{2}^{i}-1\right) \theta+\psi_{1}^{i} \sum_{l=1}^{n} \mathbb{1}\left[g_{l} \in \mathcal{I}^{i}\right] \eta_{l}+\psi_{2}^{i} \xi^{i} \\
p^{i}-p= & \left(k \psi_{1}^{i}+\psi_{2}^{i}-n \widetilde{\psi_{1}}-\widetilde{\psi_{2}}\right) \theta+\left(\psi_{1}^{i}-\widetilde{\psi_{1}}\right) \sum_{l=1}^{n} \mathbb{1}\left[g_{l} \in \mathcal{I}^{i}\right] \eta_{l} \\
& +\widetilde{\psi_{1}} \sum_{l=1}^{n} \mathbb{1}\left[g_{l} \notin \mathcal{I}^{i}\right] \eta_{l}+\psi_{2}^{i} \xi^{i} .
\end{aligned}
$$

From here, compute the loss function and take expectations to get

$$
\begin{aligned}
& -U^{i}\left(k, p^{i^{*}}(\mathcal{I}) ; p(G)\right)=(1-\alpha)\left(\left(k \psi_{1}^{i}+\psi_{2}^{i}-1\right)^{2}+\psi_{1}^{i^{2}} k \sigma_{\eta}^{2}+\psi_{2}^{i^{2}} \sigma_{\xi}^{2}\right) \\
& \quad+\alpha\left(\left(k \psi_{1}^{i}+\psi_{2}^{i}-\widetilde{n \psi_{1}}-\widetilde{\psi_{2}}\right)^{2}+\left(\psi_{1}^{i}-\widetilde{\psi_{1}}\right)^{2} k \sigma_{\eta}^{2}+\left(\widetilde{\psi_{1}}\right)^{2}(n-k) \sigma_{\eta}^{2}+\psi_{2}^{i^{2}} \sigma_{\xi}^{2}\right)+\lambda k .
\end{aligned}
$$




\section{A.4.1 Continuous Information}

Fix exogenous parameters $\hat{\sigma}_{\eta}^{2}$ and $\hat{\lambda}$, which will correspond to the precision of the authority's communication and the cost of information in the continuous model. Now, consider a sequence of model indexed by parameter $\bar{n} \rightarrow \infty$, in which the public signal noise parameter is given by $\sigma_{\eta}^{2}=\bar{n} \hat{\sigma}_{\eta}^{2}$ and the cost-per-signal is given by $\lambda=\frac{\hat{\lambda}}{\bar{n}}$. As $\bar{n}$ grows, the precision of a signal and its cost each become arbitrarily low.

The cost of information in each version of the model is invariant, in the sense that achieving a particular posterior variance on the state $\theta$ does not depend on $\bar{n}$. As an example, consider the cost of inferring the state with variance $\frac{\hat{\sigma}_{\eta}^{2}}{1+\hat{\sigma}_{\eta}^{2}}$ for a variety of $\bar{n}$. For any $\bar{n}$, doing so requires exactly $\bar{n}$ signals, since

$$
E\left[\left(E\left(\theta \mid\left\{g_{l} ; l=1, \ldots, \bar{n}\right\}\right)-\theta\right)^{2}\right]=\frac{\sigma_{\eta}^{2} / \bar{n}}{1+\sigma_{\eta}^{2} / \bar{n}}=\frac{\hat{\sigma}_{\eta}^{2}}{1+\hat{\sigma}_{\eta}^{2}}
$$

The cost of observing $\bar{n}$ signals is always $\lambda \bar{n}=\hat{\lambda}$, establishing the invariance. ${ }^{16}$

Agent $i$ 's loss function can rewritten in terms of $\hat{\sigma}_{\eta}^{2}$ and the ratios $\frac{n}{\bar{n}}$ and $\frac{k}{\bar{n}}$. The limit of this function is well-defined, so long as the limits of these ratios are also welldefined. We can now define two parameters to summarize information choices, each of which can take on a continuous (rational) value. Let $\hat{n}=\lim _{\bar{n} \rightarrow \infty} \frac{n}{\bar{n}} \in[0, \infty)$, be the information authority's choice of scope. Next, let $\hat{k}=\lim _{\bar{n} \rightarrow \infty} \frac{k}{\bar{n}}$ be agent $i$ 's information choice. Since agents can only observe those signals released by the authority, $\hat{k} \in[0, \hat{n}]$. Finally, note that cost of information can be written

\footnotetext{
${ }^{16}$ Expression (A.24) also establishes that, in terms of inference on the state, the information acquisition model here is identical to that of Myatt and Wallace (2010). That is, in terms of posterior variances of the state, it does not matter if I purchase $\bar{n}$ signals of variance $\sigma_{\eta}^{2}=\bar{n} \hat{\sigma}_{\eta}^{2}$ or a single signal of variance $\hat{\sigma}_{\eta}^{2}$. The two models have very different implications for the crosssectional correlation of information, however, which is crucial when agents interact strategically.
} 
$c(\hat{k})=\hat{\lambda} \hat{k}$.

As $\bar{n}$ become large, the absolute value of $\psi_{1}^{i}$ goes to zero. Let $\hat{\psi}_{1}^{i}=\lim _{\bar{n} \rightarrow \infty} \bar{n} \psi_{1}^{i}$ and $\hat{\psi}_{2}^{i}=\lim _{\bar{n} \rightarrow \infty} \psi_{2}^{i}$, so that

$$
\begin{aligned}
& \hat{\psi}_{1}^{i}=\frac{1}{\hat{k}+\hat{\sigma}_{\eta}^{2}+\frac{\hat{\sigma}_{\eta}^{2}}{\sigma_{\xi}^{2}}}\left((1-\alpha)+\widetilde{\hat{\psi}_{1}}\left(\hat{n}+\hat{\sigma}_{\eta}^{2}+\frac{\hat{\sigma}_{\eta}^{2}}{\sigma_{\xi}^{2}}\right)+\alpha \widetilde{\psi_{2}}\right) \\
& \hat{\psi}_{2}^{i}=\frac{1}{\hat{k}_{\frac{\sigma_{\xi}^{2}}{2}}^{2}+1+\sigma_{\xi}^{2}}\left((1-\alpha)+\alpha \widetilde{\hat{\psi}_{1}}(\hat{n}-\hat{k})+\alpha \widetilde{\psi_{2}}\right) .
\end{aligned}
$$

Finally, taking the limit of expression (A.23), agent $i$ 's welfare can now be rewritten

$$
\begin{aligned}
& -U^{i}\left(\hat{k}, p^{i^{*}}(\mathcal{I}) ; p(G)\right)=(1-\alpha)\left(\left(\hat{k} \hat{\psi}_{1}^{i}+\psi_{2}^{i}-1\right)^{2}+\left(\hat{\psi}_{1}^{i}\right)^{2} \hat{k} \hat{\sigma}_{\eta}^{2}+\left(\hat{\psi}_{2}^{i}\right)^{2} \sigma_{\xi}^{2}\right) \\
& \quad+\alpha\left(\left(\hat{k} \hat{\psi}_{1}^{i}-\hat{n} \widetilde{\hat{\psi}_{1}}\right)^{2}+\left(\hat{\psi}_{1}^{i}-\widetilde{\hat{\psi}_{1}}\right)^{2} \hat{k} \hat{\sigma}_{\eta}^{2}+{\widetilde{\hat{\psi}_{1}}}^{2}(\hat{n}-\hat{k}) \sigma_{\eta}^{2}+\left(\hat{\psi}_{2}^{i}-\widetilde{\psi_{2}}\right)^{2}+\left(\hat{\psi}_{2}^{i}\right)^{2} \sigma_{\xi}^{2}\right)+\hat{\lambda} \hat{k}
\end{aligned}
$$

Aside from the substitution of variables with ^'s, these equations are identical to their discrete counterparts in (A.19), (A.20) and (A.23). I suppress the distinction between $\hat{n}$ and $n$, etc, in the paper, but maintain it in the appendix for completeness.

\section{A.4.2 Agent Loss is Convex}

Twice-differentiating (A.27) with respect to $\hat{k}$ and simplifying substantially yields

$$
-\frac{\partial^{2} U^{i}}{\partial \hat{k}^{2}}=2 \frac{\hat{n}}{\hat{k}} \frac{\sigma_{\xi}^{2} \hat{\sigma}_{\eta}^{2}}{\widehat{\hat{\psi}}_{1}} \frac{\left(\hat{\sigma}_{\eta}^{2}+\sigma_{\xi}^{2}\left(\tilde{\hat{k}}+\hat{\sigma}_{\eta}^{2}\right)\right)^{2}}{\left(\hat{\sigma}_{\eta}^{2}+\sigma_{\xi}^{2}\left(\hat{k}+\hat{\sigma}_{\eta}^{2}\right)\right)^{3}}>0 .
$$

So agent $i$ 's loss (utility) is convex (concave) on $k \in[0, n]$. 


\section{A.4.3 Interior Levels of Acquisition}

Agent $i$ 's problem is to find

$$
\underset{\hat{k}}{\operatorname{argmax}} U^{i} \quad \text { subject to } \quad 0 \leq \hat{k} \leq \hat{n}
$$

Let $\lambda_{1}$ and $\lambda_{2}$ be the multipliers on the inequality constraints $\hat{k} \leq \hat{n}$ and $\hat{k} \geq 0$ respectively. Then the agent's first order conditions are given by

$$
\begin{aligned}
& 0=-\frac{\partial U^{i}}{\partial \hat{k}}+\lambda_{1}-\lambda_{2}+\lambda, \\
& \lambda_{1} \geq 0 ; \lambda_{2} \geq 0,
\end{aligned}
$$

and the complementary slackness conditions. A value of $\hat{k}$ that satisfies these conditions is a unique solution to the agent's optimization problem.

Differentiating agent welfare in equation (A.27) with respect to $\hat{k}$, and imposing equilibrium conditions $\tilde{\hat{k}}=\hat{k}$ yields the following expression:

$$
-\hat{\sigma}_{\eta}^{2}\left(\frac{\hat{n}}{\hat{k}} \hat{\psi}_{1}^{*}\right)^{2}+\hat{\lambda}+\lambda_{1}-\lambda_{2}=0 .
$$

For interior points, the extra Lagrange multipliers drop out to yield

$$
\hat{\lambda}=\hat{\sigma}_{\eta}^{2}\left(\frac{\hat{n}}{\hat{k}} \hat{\psi}_{1}^{*}\right)^{2}
$$

which can be solved for $\hat{k}$

$$
\ddot{\hat{k}}(\hat{n})=\frac{\left(\frac{\sigma_{\eta}^{2}}{\hat{\lambda}}\right)^{\frac{1}{2}}-\hat{\sigma}_{\eta}^{2} \tau}{1-\frac{\alpha}{\hat{n}} \hat{\sigma}_{\eta}^{2} \tau}
$$

where $\tau=\left(\frac{1}{1-\alpha}+\frac{1}{\sigma_{\xi}^{2}}\right)$. 


\section{A.4.4 Total Information Acquisition}

Full information acquisition is an equilibrium if and only if individual $i$ 's loss is (weakly) decreasing in $\hat{k}$ at $\hat{k}=\hat{n}$, when other agent's information is also full $\left(\tilde{\hat{k}}=\hat{n}\right.$.) When $\tilde{\hat{k}}=\hat{n}$, we have that $\hat{\psi}_{1}^{*}=\left(\hat{n}+\hat{\sigma}_{\eta}^{2}+(1-\alpha) \frac{\hat{\sigma}_{\eta}^{2}}{\sigma_{\xi}^{2}}\right)^{-1}$ and the required inequality is

$$
\lambda \leq \hat{\sigma}_{\eta}^{2}\left(\hat{n}+\hat{\sigma}_{\eta}^{2}+(1-\alpha) \frac{\hat{\sigma}_{\eta}^{2}}{\sigma_{\xi}^{2}}\right)^{-2} .
$$

Rearrange the inequality to show that full acquisition is an equilibrium whenever

$$
\hat{n} \leq\left(\frac{\hat{\sigma}_{\eta}^{2}}{\lambda}\right)^{\frac{1}{2}}-\sigma_{\eta}^{2}-(1-\alpha) \frac{\hat{\sigma}_{\eta}^{2}}{\sigma_{\xi}^{2}}
$$

\section{A.4.5 No Information Acquisition}

Conversely, no information acquisition is an equilibrium if and only if agent $i$ 's loss is (weakly) increasing in $\hat{k}$ ay $\hat{k}=0$, when other agents' information is also nil. Taking care to avoid dividing by zero, the agent's first order condition can be rearranged when $\tilde{\hat{k}}=0$ to yield the condition

$$
\lambda \geq \frac{\hat{\sigma}_{\eta}^{2}}{\tau^{2}\left(\hat{\sigma}_{\eta}^{2}+\hat{k} \frac{\sigma_{\xi}^{2}}{1+\sigma_{\xi}^{2}}\right)^{2}} .
$$

Evaluating at $\hat{k}=0$, the condition for no information acquisition to be an equilibrium is

$$
\lambda \geq \frac{1}{\sigma_{\eta}^{2} \tau^{2}} .
$$

Some parameter constellations satisfy both conditions (A.35) and (A.37). In this case, the model has two pure strategy equilibria. However, no interior value of 
$\hat{k}$ can simultaneously satisfy either the full information or no information conditions equation along with condition (A.32) for an interior equilbrium. By maintaining assumption 1, uniqueness is assured and the analysis is simplified.

\section{B Social Welfare Function}

In a symmetric equilibrium for a given $\hat{n}, \hat{\psi}_{1}^{i}=\frac{\hat{n}}{\hat{k}} \psi_{1}^{*}$ and $\hat{\psi}_{2}^{i}=\psi_{2}^{*}$. Evaluating equilibrium actions, social welfare can be written as

$$
\begin{gathered}
-U^{G}\left(\hat{k}, p^{i^{*}}(\mathcal{I}) ; p(G)\right)=\left(1-\alpha^{\star}\right)\left(\left(\hat{n} \hat{\psi}_{1}^{*}+\hat{\psi}_{2}^{*}-1\right)^{2}+\left(\hat{\psi}_{1}^{*} \hat{n}\right)^{2} \frac{\hat{\sigma}_{\eta}^{2}}{\hat{k}}+\left(\hat{\psi}_{2}^{*}\right)^{2} \sigma_{\xi}^{2}\right) \\
+\alpha^{\star}\left(\left(\hat{n} \hat{\psi}_{1}^{*}\right)^{2}\left(1-\frac{\hat{k}^{*}}{\hat{n}}\right)^{2} \frac{\hat{\sigma}_{\eta}^{2}}{\hat{k}}+\left(\hat{n} \hat{\psi}_{1}^{*}\right)^{2}\left(1-\frac{\hat{k}}{\hat{n}}\right) \frac{\hat{\sigma}_{\eta}^{2}}{\hat{n}}+\left(\hat{\psi}_{2}^{*}\right)^{2} \sigma_{\xi}^{2}\right)+\hat{\lambda} \hat{k} .
\end{gathered}
$$

A great deal of simplification yields the following expression

$$
-U^{G}=\hat{\sigma}_{\eta}^{2}\left(\frac{n}{k} \hat{\psi}_{1}^{*}\right)^{2}\left(\hat{k} \frac{1-\frac{\hat{k}}{\hat{n}} \alpha^{\star}}{1-\frac{\hat{k}}{\hat{n}} \alpha}+\left(1-\frac{\hat{k}}{\hat{n}} \alpha\right) \hat{\sigma}_{\eta}^{2}\left(\frac{1}{\sigma_{\xi}^{2}}+\frac{1-\alpha^{\star}}{(1-\alpha)^{2}}\right)\right)\left(1-\frac{\hat{k}}{\hat{n}} \alpha\right)+\hat{\lambda} \hat{k} .
$$

When $\alpha=\alpha^{\star}$, this is just

$$
-U^{G}=\hat{\sigma}_{\eta}^{2}\left(\frac{n}{k} \hat{\psi}_{1}^{*}\right)\left(1-\frac{\hat{k}}{\hat{n}} \alpha\right)+\hat{\lambda} \hat{k}
$$




\section{Optimal Communication: Aligned Preferences}

\section{C.1 Proof of Lemma 1}

Proof of Lemma 1. The form of $\hat{k}^{*}$ ensures that for any value $\hat{n}$ that implies $\hat{k}^{*}(\hat{n})<$ $\hat{n}$, there exists another value $\hat{n}^{\prime}<\hat{n}$, such that $\hat{k}^{*}\left(\hat{n}^{\prime}\right)=\hat{n}^{\prime}=\hat{k}^{*}(\hat{n})$. I want to show that social welfare is always higher under communication policy $\hat{n}^{\prime}$. Using the simplified expression for social welfare derived in B.3, welfare under $\hat{n}^{\prime}$ is greater if and only if

$$
\frac{\hat{k}+(1-\alpha) \sigma_{\eta}^{2} \tau}{\hat{k}+\left(1-\frac{\hat{k}^{*}}{\hat{n}} \alpha\right) \sigma_{\eta}^{2} \tau} \geq \frac{1-\alpha}{1-\frac{\hat{k}^{*}}{\hat{n}} \alpha}
$$

But, since $\frac{\hat{k}^{*}}{n}<1$ this must always be true.

\section{C.2 Optimal Scope}

Using the result of lemma 1 , I compute the optimal choice of $\hat{k}$ assuming that $\hat{k}=\hat{n}$. I then confirm that, under the implied policy, agents do choose $\hat{k}^{*}=\hat{n}$. If so, this represents the optimal level of transparency.

Under full acquisition, the social planner seeks to minimize loss given by

$$
-U^{G}=\left(1-\alpha^{\star}\right)\left[\left(\hat{n} \hat{\psi}_{1}^{*}+\hat{\psi}_{2}^{*}-1\right)^{2}+\left(\hat{\psi}_{1}^{*}\right)^{2} \hat{n} \hat{\sigma}_{\eta}^{2}\right]+\left(\hat{\psi}_{2}^{*}\right)^{2} \sigma_{\xi}^{2}+\lambda \hat{n} .
$$

The first order condition is

$$
-\left(1-\alpha^{\star}\right) \hat{\sigma}_{\eta}^{2}\left(\hat{\psi}_{1}^{*}\right)^{2}+2\left(\hat{\psi}_{1}^{*}\right)^{3}\left(\hat{\sigma}_{\eta}^{2}\right)^{2} \frac{1-\alpha}{\sigma_{\xi}^{2}}\left(\alpha-\alpha^{\star}\right)+\lambda=0 .
$$


When preferences are aligned, this reduces to

$$
(1-\alpha) \hat{\sigma}_{\eta}^{2}\left(\hat{\psi}_{1}^{*}\right)^{2}=\lambda
$$

which can be solved for $\hat{n}^{\star}$ :

$$
\hat{n}^{\star}=\sqrt{\frac{(1-\alpha) \hat{\sigma}_{\eta}^{2}}{\lambda}}-\hat{\sigma}_{\eta}^{2}-(1-\alpha) \frac{\hat{\sigma}_{\eta}^{2}}{\sigma_{\xi}^{2}} .
$$

Inspection shows that this is less than the threshold value $\widehat{\hat{n}}$, and so the result is established.

\section{Constraints on Authority's Information}

\section{D.1 Equilibrium Actions}

Suppose $\sigma_{\varepsilon}^{2}>0$. Then, the conditional expectation of signal $l$ is given by

$$
E\left(g_{l} \mid \mathcal{I}^{i}\right)= \begin{cases}g_{l} & \text { if } g_{l} \in \mathcal{I}^{i} \\ E\left(\theta+\varepsilon \mid \mathcal{I}^{i}\right) & \text { if } g_{l} \notin \mathcal{I}^{i}\end{cases}
$$

Agent $i$ 's conditional expectation of the state and of the authority's "mistake" are now given respectively by

$$
\begin{aligned}
& E\left(\theta \mid \mathcal{I}^{i}\right)=\gamma_{1} \sum_{l=1}^{n} \mathbb{1}\left[g_{l} \in \mathcal{I}^{i}\right] g_{l}+\gamma_{2} r^{i} \\
& E\left(\varepsilon \mid \mathcal{I}^{i}\right)=a_{1} \sum_{l=1}^{n} \mathbb{1}\left[g_{l} \in \mathcal{I}^{i}\right] g_{l}+a_{2} r^{i}
\end{aligned}
$$


where $\gamma_{1}=\frac{\sigma_{\xi}^{2}}{\chi+\sigma_{\xi}^{2}(k+\chi)}, \gamma_{2}=\frac{\chi}{\chi+\sigma_{\xi}^{2}(k+\chi)}, a_{1}=\frac{\sigma_{\xi}^{2}\left(1+\sigma_{\xi}^{2}\right)}{\chi+\sigma_{\xi}^{2}(k+\chi)}, a_{2}=-\frac{k \sigma_{\xi}^{2}}{\chi+\sigma_{\xi}^{2}(k+\chi)}$, and $\chi=k \sigma_{\varepsilon}^{2}+\sigma_{\eta}^{2}$.

Individual $i$ 's action is given by

$$
\begin{aligned}
p^{i}= & {\left[(1-\alpha) \gamma_{1}+\alpha\left(\psi_{1}\left(1+(n-k)\left(\gamma_{1}+a_{1}\right)\right)+\psi_{2} \gamma_{1}\right)\right] \sum_{l=1}^{n} \mathbb{1}\left[g_{l} \in \mathcal{I}^{i}\right] g_{l} } \\
& +\left[(1-\alpha) \gamma_{2}+\alpha\left(\psi_{1}(n-k)\left(\gamma_{2}+a_{2}\right)+\psi_{2} \gamma_{2}\right)\right] r^{i}
\end{aligned}
$$

Once again computing the expectation of the aggregate action gives

$$
\begin{aligned}
E\left(p \mid \mathcal{I}^{i}\right)= & \left(\psi_{1}\left(1+(n-k)\left(\gamma_{1}+a_{1}\right)\right)+\psi_{2} \gamma_{1}\right) \sum_{l=1}^{n} \mathbb{1}\left[g_{l} \in \mathcal{I}^{i}\right] g_{l} \\
& +\left(\psi_{1}(n-k)\left(\gamma_{2}+a_{2}\right)+\psi_{2} \gamma_{2}\right) r^{i}
\end{aligned}
$$

Finding the fixed point as before yields the equilibrium coefficients,

$$
\begin{aligned}
& \psi_{1}^{*}=\left(n \Gamma+\left(\frac{n}{k}-\alpha\right) \sigma_{\eta}^{2}\left(\frac{1}{1-\alpha}+\frac{1}{\sigma_{\xi}^{2}}\right)\right)^{-1} \\
& \psi_{2}^{*}=\left(\Gamma+\sigma_{\xi}^{2}\left(\frac{1}{1-\alpha}+\frac{k}{(1-\alpha) k \sigma_{\varepsilon}^{2}+\left(1-\alpha \frac{k}{n}\right) \frac{1}{\sigma_{\eta}^{2}}}\right)\right)^{-1},
\end{aligned}
$$

where $\Gamma \equiv 1+\sigma_{\varepsilon}^{2}+(1-\alpha) \frac{\sigma_{\varepsilon}^{2}}{\sigma_{\xi}^{2}}$.

\section{D.2 Equilibrium Information}

The agent's problem is exactly as in A.4.3 and the first order conditions are identical (up the relevant definition of $\hat{\psi}_{1}^{*}$.)

$$
-\hat{\sigma}_{\eta}^{2}\left(\frac{n}{k} \hat{\psi}_{1}^{*}\right)^{2}+\lambda+\lambda_{1}-\lambda_{2}=0
$$


The derivations of equilibrium information follow exactly as before. One potentially surprising result is that the condition for zero information to be an equilibrium does not change. That is, there is no need to restate assumption 1 as long $\sigma_{\varepsilon}^{2}<\infty$.

\section{D.3 Choice of Scope}

Social welfare under the assumption $\hat{k}=\hat{n}$ is now written

$$
U^{G}=(1-\alpha)\left[\left(\hat{n} \hat{\psi}_{1}^{*}+\hat{\psi}_{2}^{*}-1\right)^{2}+\left(\hat{\psi}_{1}^{*}\right)^{2} \hat{n} \hat{\sigma}_{\eta}^{2}+\left(\hat{n} \hat{\psi}_{1}^{*}\right)^{2} \sigma_{\varepsilon}^{2}\right]+\left(\hat{\psi}_{2}^{*}\right)^{2} \sigma_{\xi}^{2}+\lambda \hat{n}
$$

Taking the first order condition and solving for $\hat{n}^{\star}$ yields

$$
\hat{n}^{\star}=\left(\sqrt{\frac{\hat{\sigma}_{\eta}^{2}(1-\alpha)}{\hat{\lambda}}}-(1-\alpha) \hat{\sigma}_{\eta}^{2} \tau\right) \frac{1}{\Gamma} .
$$

\section{E Optimal Communication: Misaligned Prefer- ences}

\section{E.1 Private Information}

Proof of Proposition $4(c)$. Suppose $\hat{\sigma}_{\eta}^{2}$ is given. Then, when $\alpha \leq \alpha^{\star}$, the authority always prefers that agents acquire full information. To see this compare social welfare for a given $\hat{k}$, for the case that $\hat{k}<\hat{n}$ and the case that $\hat{k}=\hat{n}$. In many cases, full acquisition may also be optimal when $\alpha>\alpha^{\star}$ as well. In these cases, it follows directly that $\hat{n}^{\star}$ is finite.

Now suppose $\alpha>\alpha^{\star}$ and the the authority finds it optimal to select $\hat{n}^{o}>\widehat{\hat{n}}$. To find $\hat{n}^{o}$ in this case, plug in agents' information acquisition $\ddot{k}(\hat{n})$, take the first order 
condition with respect to $\hat{n}$, and solve. This first order condition has a unique (real) solution, $\hat{n}^{o}$, and it is finite. To show that this is indeed an optimum, however, requires slightly more work. Taking second order condition and evaluating at $\hat{n}^{o}$ shows that the loss function is locally convex, and since there is only one critical point, that this is indeed the global optimum.

The general expression for $\hat{n}^{o}$ is unwieldy. However, when $\alpha^{\star}=0$ it simplifies substantially to

$$
\hat{n}^{o}=(1-\alpha)^{2}\left(\sqrt{\frac{\hat{\sigma}_{\eta}^{2}}{\hat{\lambda}}}\left(\frac{1}{(1-\alpha)^{2}}+\frac{1}{\sigma_{\xi}^{2}}\right)-\hat{\sigma}_{\eta}^{2} \tau^{2}\right)
$$

Proof of Proposition 5(c). It suffices to show that welfare is improving in $\frac{1}{\hat{\sigma}_{\eta}^{2}}$, given optimal scope, for any level $\hat{\sigma}_{\eta}^{2}$. If optimal scope calls for full acquisition, the authority can always decrease scope to maintain the same total precision and benefit from lower information costs. I now demonstrate that when optimal scope lies to the right $\widehat{\hat{n}}$, welfare is again improving if scope is adjusted optimally. Substitute in $\ddot{k}(\hat{n})$, and then $\hat{n}^{o}$, into the social welfare function given in (B.2). The derivative with respect to $\hat{\sigma}_{\eta}^{2}$ is greater than zero by assumption 1 , and the result is established.

\section{F Directed Search}

In this section, I solve a version of the model with a more general type of information choice, in which agents may choose the probability with which they observe particular signals, but at a cost. The logic parallels that of section A.2, 
despite some added complications.

Assume now that agents assign relative weights $w_{1}, w_{2}, \ldots, w_{n}$ to each signal released by the information authority, so that the probability of drawing the $j$ 'th signal as the first signal drawn is $\frac{w_{j}}{\sum_{l=1}^{n} w_{l}}$. I again assume that signals are drawn sequentially, without replacement. Because signals are drawn without replacement, the probability that $g_{j}$ is drawn on the second draw depends on which signal was drawn in the first round, and so on. The distribution characterizing this search process is know as the generalized Wallenius noncentral hypergeometric distribution.

Let $\mu_{l}=P\left(\mathcal{G}_{l}^{i}=1 ;\left\|\mathcal{G}^{i}\right\|=k\right)$ be the probability that signal $l$ is drawn among a sample of $k$ signals. Unfortunately, for $k>1$ there is no requirement that $\mu_{l}$ is proportional to $w_{l}$ and, in fact, there is no closed-form solution for $\mu_{l}$ as a function of the $w_{l}$ 's. Chen et al. (1994) show, however, that a set of $w_{l}$ 's can be mapped unquietly into a set of $\mu_{l}$ 's and the two respect a natural ordering relation

$$
w_{l}>w_{j} \Longleftrightarrow \mu_{l}>\mu_{j} .
$$

To simplify the analysis, and because the agents care directly about $\mu_{l}$, I proceed as if these are the fundamental choice of the agents, although they could always be mapped back into a set of weights used for the sampling process. The $\mu_{l}$ 's also have the important property that $\sum_{l=1}^{n} \mu_{l}=k$.

\section{F.1 Equilibrium Actions}

The equilibrium pricing rule must reflect the fact that some signals may, in general, be observed by more agents than others. Therefore I guess the following 
form for a linear equilibrium

$$
p=\sum_{l=1}^{n} \tilde{\psi}_{l} g_{l}
$$

Under the baseline information assumptions, we have that

$$
\begin{gathered}
E\left(\theta \mid \mathcal{I}^{i}\right)=\frac{1}{k+\sigma_{\eta}^{2}} \sum_{l=1}^{n} \mathbb{1}\left[g_{l} \in \mathcal{I}^{i}\right] g_{l} \\
E\left(g_{l} \mid \mathcal{I}^{i}\right)= \begin{cases}g_{l} & \text { if } g_{l} \in \mathcal{I}^{i} \\
E\left(\theta \mid \mathcal{I}^{i}\right) & \text { if } g_{l} \notin \mathcal{I}^{i} .\end{cases}
\end{gathered}
$$

Optimal action on the part of agent $i$ implies

$$
\begin{aligned}
p^{i} & =\frac{(1-\alpha)}{k+\sigma_{\eta}^{2}} \sum_{l=1}^{n} \mathbb{1}\left[g_{l} \in \mathcal{I}^{i}\right] g_{l}+\alpha \sum_{l=1}^{n} \tilde{\psi}_{l}\left[\mathbb{1}\left[g_{l} \in \mathcal{I}^{i}\right] g_{l}+\mathbb{1}\left[g_{l} \notin \mathcal{I}^{i}\right] \frac{1}{k+\sigma_{\eta}^{2}} \sum_{j=1}^{n} \mathbb{1}\left[g_{j} \in \mathcal{I}^{i}\right] g_{j}\right] \\
& =\sum_{l=1}^{n}\left[\frac{1-\alpha}{k+\sigma_{\eta}^{2}}+\alpha \tilde{\psi}_{l}+\frac{\alpha}{k+\sigma_{\eta}^{2}} \sum_{j=1}^{n} \tilde{\psi}_{j} \mathbb{1}\left[g_{j} \notin \mathcal{I}^{i}\right]\right] \mathbb{1}\left[g_{l} \in \mathcal{I}^{i}\right] g_{l} \\
& \equiv \sum_{l=1}^{n} \hat{\psi}_{l}^{i} \mathbb{1}\left[g_{l} \in \mathcal{I}^{i}\right] g_{l} .
\end{aligned}
$$

where $\hat{\psi}_{l}^{i}$ is agent $i$ 's optimal response to signal $l$ conditional on $g_{l} \in \mathcal{I}^{i}$.

At this point, a new complication arises in that $\hat{\psi}_{l}^{i}$ is a random variable, both cross sectionally and from the perspective of agent $i$. This randomness is problematic because $\hat{\psi}_{l}^{i}$ and $\mathbb{1}\left[g_{l} \in \mathcal{I}^{i}\right]$ are not independent and no closed form expression exists for their covariance. This complicates the step of integrating across agents in order to determine the aggregate action rule. The key observation required to circumvent this difficulty is that, as $\bar{n}$ grows larger, $\hat{\psi}_{l}^{i}$ becomes essentially deterministic. This allows for both straightforward aggregation across agents and simple computation of expected values. 
To make this claim more precise, consider once again a sequence of models indexed by $\bar{n}$, in which $\lim _{\bar{n} \rightarrow \infty} \frac{n}{\bar{n}}=\hat{n}$ and $\lim _{\bar{n} \rightarrow \infty} \frac{k}{\bar{n}}=\hat{k}$, and $\sigma_{\eta}^{2}=\bar{n} \hat{\sigma}_{\eta}^{2}$. Define the set of random variables $x_{j}=n \widetilde{\psi}_{\widetilde{l}_{j}} \mathbb{1}\left[g_{\widetilde{l}_{j}} \notin \mathcal{I}^{i}\right]$, where the indexes $\widetilde{l}_{j}, j=1,2, \ldots, n$, are generated by randomly drawing an index $l$, without replacement, from among the $n$ public signals. Define

$$
\begin{aligned}
& b_{\bar{n}} \equiv E\left[x_{1}\right]=\frac{1}{n} \sum_{l=1}^{n} n \widetilde{\psi}_{l}\left(1-\mu_{l}\right) \\
& \delta_{\bar{n}} \equiv \frac{1}{n} \sum_{j=1}^{n} n \widetilde{\psi}_{l} \mathbb{1}\left[g_{j} \notin \mathcal{I}^{i}\right]-b_{\bar{n}}=\frac{1}{n} \sum_{j=1}^{n} x_{j}-b_{\bar{n}} .
\end{aligned}
$$

Then, equation (F.5) can then be rewritten after some manipulation as

$$
p^{i}=\sum_{l=1}^{n}\left[\frac{1-\alpha}{k+\sigma_{\eta}^{2}}+\alpha \widetilde{\psi}_{l}+\frac{\alpha}{k+\sigma_{\eta}^{2}}\left(b_{\bar{n}}+\delta_{\bar{n}}\right)\right] \mathbb{1}\left[g_{l} \in \mathcal{I}^{i}\right] g_{l} .
$$

Integrate across agents to get

$$
p=\sum_{l=1}^{n}\left(\frac{\alpha}{k+\sigma_{\eta}^{2}} \Delta_{\bar{n}, l}+\mu_{l}\left[\frac{1-\alpha}{k+\sigma_{\eta}^{2}}+\alpha \widetilde{\psi}_{l}+\frac{\alpha}{k+\sigma_{\eta}^{2}} b_{\bar{n}}\right]\right) g_{l}
$$

where $\Delta_{\bar{n}, l} \equiv E\left[\delta_{\bar{n}} \mathbb{1}\left[g_{l} \in \mathcal{I}^{i}\right]\right]$. The equilibrium coefficients are then given by the fixed point of the expression

$$
\widetilde{\psi}_{l}=\frac{\alpha}{k+\sigma_{\eta}^{2}} \Delta_{\bar{n}, l}+\mu_{l}\left[\frac{1-\alpha}{k+\sigma_{\eta}^{2}}+\alpha \widetilde{\psi}_{l}+\frac{\alpha}{k+\sigma_{\eta}^{2}} b_{\bar{n}}\right] .
$$

Now, solving for $\widetilde{\psi}_{l}$ yields

$$
\widetilde{\psi}_{l}=\frac{\mu_{l}}{1-\alpha \mu_{l}} \frac{1}{k+\sigma_{\eta}^{2}}\left(1-\alpha+\alpha b_{\bar{n}}\right)+\frac{\alpha}{\left(1-\alpha \mu_{l}\right)\left(k+\sigma_{\eta}^{2}\right)} \Delta_{\bar{n}, l}
$$


Now, using the fact that $-E\left(\left|\delta_{\bar{n}}\right|\right) \leq \Delta_{\bar{n}, l} \leq E\left(\left|\delta_{\bar{n}}\right|\right)$, we have the inequality

$$
\widetilde{\psi}_{l} \leq \frac{\mu_{l}}{1-\alpha \mu_{l}} \frac{1}{k+\sigma_{\eta}^{2}}\left(1-\alpha+\alpha b_{\bar{n}}\right)+\frac{\alpha}{\left(1-\alpha \mu_{l}\right)\left(k+\sigma_{\eta}^{2}\right)} E\left(\left|\delta_{\bar{n}}\right|\right)
$$

and a corresponding lower bound on $\widetilde{\psi}_{l}$. Substituting recursively and simplifying yields the following bounds on $\widetilde{\psi}_{l}$

$$
\frac{\mu_{l}}{1-\alpha \mu_{l}} \rho_{1}-E\left(\left|\delta_{\bar{n}}\right|\right) \frac{\alpha}{\left(1-\alpha \mu_{l}\right)} \rho_{2, l} \leq \widetilde{\psi}_{l} \leq \frac{\mu_{l}}{1-\alpha \mu_{l}} \rho_{1}+E\left(\left|\delta_{\bar{n}}\right|\right) \frac{\alpha}{\left(1-\alpha \mu_{l}\right)} \rho_{2, l}
$$

where

$$
\begin{aligned}
\rho_{1} & =\frac{1-\alpha}{k+\sigma_{\eta}^{2}-\alpha q} \\
\rho_{2, k} & =\left(\frac{1}{k+\sigma_{\eta}^{2}}+\frac{\alpha \mu_{l} \bar{q}}{\left(k+\sigma_{\eta}^{2}\right)\left(k+\sigma_{\eta}^{2}-\alpha q\right)}\right) \\
q & =\sum_{l=1}^{n} \frac{\left(1-\mu_{l}\right) \mu_{l}}{1-\alpha \mu_{l}} \\
\bar{q} & =\sum_{l=1}^{n} \frac{\left(1-\mu_{l}\right)}{1-\alpha \mu_{l}} .
\end{aligned}
$$

Now, multiply the inequality by $\bar{n}$, to get

$\frac{\mu_{l}}{1-\alpha \mu_{l}} \bar{n} \rho_{1}-E\left(\left|\delta_{\bar{n}}\right|\right) \frac{\alpha}{\left(1-\alpha \mu_{l}\right)} \bar{n} \rho_{2, k} \leq \bar{n} \widetilde{\psi}_{l} \leq \frac{\mu_{l}}{1-\alpha \mu_{l}} \bar{n} \rho_{1}+E\left(\left|\delta_{\bar{n}}\right|\right) \frac{\alpha}{\left(1-\alpha \mu_{l}\right)} \bar{n} \rho_{2, k}$.

A law of large numbers applies to $\frac{1}{n} \sum_{j=1}^{n} x_{j}$, implying that $\lim _{\bar{n} \rightarrow \infty} E\left(\left|\delta_{\bar{n}}\right|\right)=$ 
$0 .{ }^{17}$ Let

$$
Q \equiv \lim _{\bar{n} \rightarrow \infty} \frac{1}{\bar{n}} \sum_{l=1}^{n} \frac{\left(1-\mu_{l}\right) \mu_{l}}{1-\alpha \mu_{l}} .
$$

This is clearly finite, since each term in the summand is positive and bounded by a finite constant, while the total is divided by $n$. For the same reasons, $\bar{Q} \equiv$ $\lim _{\bar{n} \rightarrow \infty} \frac{1}{\bar{n}} \sum_{l=1}^{n} \frac{1-\mu_{l}}{1-\alpha \mu_{l}}$ is also finite. Therefore, $\bar{n} \rho_{1}$ and $\bar{n} \rho_{2, l}$ each converge to a finite values and we can conclude that

$$
\begin{array}{ll}
\lim _{\bar{n} \rightarrow \infty} \bar{n} \widetilde{\psi}_{l}=\frac{\mu_{l}}{1-\alpha \mu_{l}} \rho & \equiv \widetilde{\varphi}(l) \\
\lim _{\bar{n} \rightarrow \infty} \bar{n} \hat{\psi}_{l}=\frac{1-\alpha}{\hat{k}+\hat{\sigma}_{\eta}^{2}}+\alpha \widetilde{\varphi}_{l}+\frac{\alpha}{\hat{k}+\hat{\sigma}_{\eta}^{2}} \sum_{k=0}^{\infty}\left(1-\mu_{l}\right) \widetilde{\varphi}_{l} & \equiv \varphi(l),
\end{array}
$$

where $\rho=\frac{1-\alpha}{k+\hat{\sigma}_{\eta}^{2}-\alpha Q}$.

\section{F.2 Agents' Information Choice}

I now follow a similar strategy to compute the loss of agent $i$, taking aggregate actions as given. To begin, compute the deviations

$$
\begin{aligned}
& p^{i}-\theta=\left(\sum_{l=1}^{n} \hat{\psi}_{l} \mathbb{1}\left[g_{l} \in \mathcal{I}^{i}\right]-1\right) \theta+\sum_{l=1}^{n} \hat{\psi}_{l} \mathbb{1}\left[g_{l} \in \mathcal{I}^{i}\right] \eta_{l} \\
& p^{i}-p=\left(\sum_{l=1}^{n} \hat{\psi}_{l} \mathbb{1}\left[g_{l} \in \mathcal{I}^{i}\right]-\widetilde{\psi}_{l}\right) \theta+\sum_{l=1}^{n}\left(\hat{\psi}_{l}-\widetilde{\psi}_{l}\right) \mathbb{1}\left[g_{l} \in \mathcal{I}^{i}\right] \eta_{l}+\sum_{l=1}^{n} \widetilde{\psi}_{l} \mathbb{1}\left[g_{l} \notin \mathcal{I}^{i}\right] \eta_{l}
\end{aligned}
$$

of the discrete model. We are interested in computing $E\left[\left(p^{i}-\theta\right)^{2}\right]$ and $E\left[\left(p^{i}-p\right)^{2}\right]$.

\footnotetext{
${ }^{17}$ This follows from the construction of $x_{j}$ as a sequence of exchangeable random variables. See McCall (1991) for a detailed discussion and additional references on the topic of exchangeability.
} 
First, consider the "fundamental deviation" given by

$$
\begin{aligned}
E\left[\left(p^{i}-\theta\right)^{2}\right] & =E\left(\sum_{l=1}^{n} \hat{\psi}_{l} \mathbb{1}\left[g_{l} \in \mathcal{I}^{i}\right]-1\right)^{2}+\sum_{l=1}^{n} E\left(\hat{\psi}_{l} \mathbb{1}\left[g_{l} \in \mathcal{I}^{i}\right]\right)^{2} \sigma_{\eta}^{2} \\
& =E\left(\frac{1}{\bar{n}} \sum_{l=1}^{n} \bar{n} \hat{\psi}_{l} \mathbb{1}\left[g_{l} \in \mathcal{I}^{i}\right]-1\right)^{2}+\frac{1}{\bar{n}} \sum_{l=1}^{n} E\left(\bar{n} \hat{\psi}_{l} \mathbb{1}\left[g_{l} \in \mathcal{I}^{i}\right]\right)^{2} \frac{\sigma_{\eta}^{2}}{\bar{n}} \\
& =E\left[\left(\frac{1}{\bar{n}} \sum_{l=1}^{n} \bar{n} \hat{\psi}_{l} \mathbb{1}\left[g_{l} \in \mathcal{I}^{i}\right]\right)^{2}-2 \frac{1}{\bar{n}} \sum_{l=1}^{n} \bar{n}_{l} \mathbb{1}\left[g_{l} \in \mathcal{I}^{i}\right]+1\right] \\
& +\frac{1}{\bar{n}} \sum_{l=1}^{n} E\left(\bar{n} \hat{\psi}_{l} \mathbb{1}\left[g_{l} \in \mathcal{I}^{i}\right]\right)^{2} \frac{\sigma_{\eta}^{2}}{\bar{n}} .
\end{aligned}
$$

Taking the limit $\bar{n} \rightarrow \infty$ and rewriting the infinite sum as an integral over the domain $l \in[0, \hat{n}]$, expression (F.20) now simplifies considerably to

$$
E\left[\left(p^{i}-\theta\right)^{2}\right]=\left(\int_{o}^{\hat{n}} \mu(l) \varphi(l) d l-1\right)^{2}+\hat{\sigma}_{\eta}^{2} \int_{0}^{\hat{n}} \mu(l) \varphi(l)^{2} d l .
$$

The limiting "coordination loss" term can be derived in the same manner:

$$
\begin{aligned}
E\left[\left(p^{i}-p\right)^{2}\right]= & \left(\int_{0}^{\hat{n}} \mu(l)(\varphi(l)-\widetilde{\varphi}(l)) d l\right)^{2}+\hat{\sigma}_{\eta}^{2} \int_{0}^{\hat{n}} \mu(l)(\varphi(l)-\widetilde{\varphi}(l))^{2} d l \\
& +\hat{\sigma}_{\eta}^{2} \int_{0}^{\hat{n}}(1-\mu(l)) \widetilde{\varphi}(l)^{2} d l .
\end{aligned}
$$

Finally, write the cost of information as the functional mapping $\mu(l)$ to the cost 
$c(\mu(l))$. Now, combining all terms yields agent $i$ 's welfare function

$$
\begin{aligned}
-U^{i}= & (1-\alpha)\left[\left(\int_{0}^{\hat{n}} \mu(l) \varphi(l) d l-1\right)^{2}+\hat{\sigma}_{\eta}^{2} \int_{0}^{\hat{n}} \mu(l) \varphi(l)^{2} d l\right] \\
& +\alpha\left[\left(\int_{0}^{\hat{n}}(\mu(l) \varphi(l)-\widetilde{\varphi}(l)) d l\right)^{2}+\hat{\sigma}_{\eta}^{2} \int_{0}^{\hat{n}} \mu(l)(\varphi(l)-\widetilde{\varphi}(l))^{2} d l\right. \\
& \left.+\hat{\sigma}_{\eta}^{2} \int_{0}^{\hat{n}}(1-\mu(l)) \widetilde{\varphi}(l)^{2} d l\right]+c(\mu(l)) .
\end{aligned}
$$

The solution to agent $i$ 's problem is characterized by

$$
\underset{\mu(l), \hat{k}}{\operatorname{argmax}} U^{i} \quad \text { subject to } \quad \mu(l) \leq 1 ; \mu(l) \geq 0 ; \int_{0}^{\hat{n}} \mu(l) d l \leq \hat{k} .
$$

Let $\lambda_{1}(l), \lambda_{2}(l), \lambda_{3}$ be Lagrange multipliers on the three constraints respectively.

\section{F.2.1 Equilibrium Characterization}

Taking derivatives with respect to $\mu(l)$ and $\hat{k}$ yields

$$
\begin{aligned}
0 & =2(1-\alpha)\left[(\phi-1)\left(\varphi(l)-\frac{\alpha \hat{k}}{\hat{k}+\hat{\sigma}_{\eta}^{2}} \widetilde{\varphi}(l)\right)+\hat{\sigma}_{\eta}^{2}\left(\frac{\varphi^{2}(l)}{2}-\frac{\alpha \widetilde{\varphi}(l)}{\hat{k}+\hat{\sigma}_{\eta}^{2}} \int_{0}^{\hat{n}} \mu(j) \varphi(j) d j\right)\right] \\
& +2 \alpha\left[\left(\phi-\int_{0}^{\hat{n}} \widetilde{\varphi}(j) d j\right)\left(\varphi(l)-\frac{\alpha \hat{k}}{\hat{k}+\hat{\sigma}_{\eta}^{2}} \widetilde{\varphi}(l)\right)+\hat{\sigma}_{\eta}^{2}\left(\frac{\varphi(l)^{2}}{2}-\varphi(l) \widetilde{\varphi}(l)\right.\right. \\
& \left.\left.-\widetilde{\varphi}(l) \frac{\alpha}{\hat{k}+\hat{\sigma}_{\eta}^{2}} \int_{0}^{\hat{n}} \mu(j)(\varphi(j)-\widetilde{\varphi}(j)) d j\right)\right]+\lambda_{1}(l)-\lambda_{2}(l)+\lambda_{3}+c_{l}(\mu(l)) \\
-\lambda_{3} & =2(1-\alpha) \frac{\partial \varphi}{\partial \hat{k}}\left[(\phi-1) \int_{0}^{\hat{n}} \mu(j) d j+\hat{\sigma}_{\eta}^{2} \int_{0}^{\hat{n}} \mu(j) \varphi(j) d j\right] \\
& +2 \alpha \frac{\partial \varphi}{\partial \hat{k}}\left[\left(\phi-\int_{0}^{\hat{n}} \widetilde{\varphi}(j) d j\right) \int_{0}^{\hat{n}} \mu(j) d j+\hat{\sigma}_{\eta}^{2} \int_{0}^{\hat{n}} \mu(j)(\varphi(j)-\widetilde{\varphi}(j)) d j\right],
\end{aligned}
$$


where $\phi=\int_{0}^{\hat{n}} \mu(l) \varphi(l) d l$ and

$$
\frac{\partial \varphi}{\partial \hat{k}} \equiv-\left(\frac{1}{\hat{k}+\hat{\sigma}_{\eta}^{2}}\right)^{2}\left((1-\alpha)+\alpha \int_{0}^{\hat{n}}(1-\mu(j)) \widetilde{\varphi}(j) d j\right)=\frac{\partial \varphi(l)}{\partial \hat{k}}
$$

is constant across $l$.

Substituting the equilibrium relationships

$$
\begin{aligned}
\hat{k} & =\int_{0}^{\hat{n}} \mu(j) d j \\
\widetilde{\varphi}(l) & =\mu(l) \varphi \\
\varphi & =\left(\frac{1}{1-\alpha \mu(l)}\right) \rho
\end{aligned}
$$

into equation (F.24) and simplifying substantially yields

$2(1-\alpha) \rho(\phi-1)+\hat{\sigma}_{\eta}^{2} \rho^{2} \frac{2 \alpha^{2} \mu(l)^{2}-4 \alpha \mu(l)+1}{(1-\alpha \mu(l))^{2}}+\lambda_{1}(l)-\lambda_{2}(l)+\lambda_{3}+c_{l}(\mu(l))=0$.

Additional algebra shows that $(\phi-1)=-\frac{1}{\hat{k}+\hat{\sigma}_{\eta}^{2}-\alpha Q} \hat{\sigma}_{\eta}^{2}$. Using this result, the first order condition simplifies further to

$$
-\hat{\sigma}_{\eta}^{2}\left(\frac{\rho}{1-\alpha \mu(l)}\right)^{2}+\lambda_{1}(l)-\lambda_{2}(l)+\lambda_{3}+c_{l}(\mu(l))=0 .
$$

Furthermore, algebraic manipulations of (F.25) establishes that in equilibrium $\lambda_{3}=$ 0

Let $\bar{\lambda}_{1}(l)=\lambda_{1}(l)(1-\alpha \mu(l))^{2}$ and $\bar{\lambda}_{2}(l)=\lambda_{2}(l)(1-\alpha \mu(l))^{2}$. Proposition 8 combines the above results to characterize the set of equilibria in the extended model. 
Proposition 8. The set of equilibria in the model are characterized by 1) the set of equalities indexed by $l$,

$$
c_{l}(\mu(l))(1-\alpha \mu(l))^{2}=\hat{\sigma}_{\eta}^{2} \rho^{2}-\bar{\lambda}_{1}(l)+\bar{\lambda}_{2}(l)
$$

the inequality constraints

$$
\mu(l) \leq 1 ; \mu(l) \geq 0 ; \int_{0}^{\hat{n}} \mu(l) d l \leq \hat{k}
$$

the complementarity slackness conditions, $\bar{\lambda}_{1}(l)(\mu(l)-1)=0, \bar{\lambda}_{2}(l) \mu=0$, and the inequalities, $\bar{\lambda}_{1}(l) \geq 0, \bar{\lambda}_{2}(l) \geq 0$.

\section{F.3 A Sufficient Condition for Uniqueness}

Suppose that the cost the information is given by the CES aggregator in equation (4.2), with $\omega>1$. The derivative of cost with respect to $\mu(l)$ is

$$
c_{l}(\mu(l))=\lambda \hat{n}^{\frac{\omega-1}{\omega}}\left(\int_{0}^{\hat{n}} \mu(l)^{\omega} d l\right)^{\frac{1-\omega}{\omega}} \mu(l)^{\omega-1} .
$$

The model has a unique equilibrium whenever the left-hand side of (F.31) is monotonically increasing in $\mu(l)$. To see this, note first that one can immediately rule out $\mu(l)=0$, since the derivative of the cost function with respect to $\mu(l)$ is always zero when $\mu(l)=0$. Second, note that if $\mu(l) \in(0,1)$ satisfies

$$
c_{l}(\mu(l))\left(1-\alpha \mu_{l}\right)^{2}=\hat{\sigma}_{\eta}^{2} \rho^{2}
$$

for any $k$, then monotonicity implies that $c_{l}(\mu(l))\left(1-\alpha \mu_{l}\right)^{2}>\hat{\sigma}_{\eta}^{2} \rho^{2}$ at $\mu(l)=1$, 
ruling out the possibility that $\lambda_{1}(l) \geq 0$, and therefore that $\mu(l)=1$, for any $k$. Finally, when $\bar{\lambda}_{1}(l)=\bar{\lambda}_{2}(l)=0$ and the lefthand side is monotonic, only one value $\mu(l)$ can simultaneously satisfy equation (F.31), so that $\mu(l)=\nu$ and the equilibrium conditions reduce to the those from the baseline model.

The required monotonicity is achieved whenever

$$
\mu(l)^{\omega-1}(1-\alpha \mu(l))^{2}
$$

is monotonic on $[0,1]$. Taking a derivative and imposing the inequality quickly establishes the requirement that

$$
\omega>\frac{1+\alpha}{1-\alpha}
$$

\section{F.4 Multiple Equilibria when Information Cost is Linear}

Suppose now that that the derivative of the cost function $c_{l}(\mu(l))=\hat{\lambda}$. An immediate implication of proposition 8 is that, in equilibrium, the function $\mu(l)$ can take on no more than one interior value, in addition to $\mu(l)=0$ or $\mu(l)=1$. To see this, consider expression (F.31) for a value of $l$ for which neither constraint one nor constraint two is binding. In this case, the left-hand side of 8 is strictly decreasing in $\mu(l)$, implying that no more than one interior value of $\mu(l)$ can simultaneously satisfy the equation. In contrast to case the case where the left hand side is

increasing, however, it still may be that $\bar{\lambda}_{1}(l)>0$ or $\bar{\lambda}_{2}(l)>0$ or both, creating the potential for a great deal of multiplicity.

Imposing the restriction that $\mu(l)$ take on no more than one interior value, a set of simple conditions can be derived characterizing the set of equilibria in the 
model. Let $\hat{n}_{1}, \hat{n}_{2}, \hat{n}_{3} ; \hat{n} \geq \hat{n}_{i} \geq 0 ; \hat{n}=\sum_{i=1}^{3} \hat{n}_{i}$ denote the "mass" of signals taking on values $\mu^{*} \in(0,1), \bar{\mu}=1, \underline{\mu}=0$, respectively. Solving the first order condition for $\mu^{*}$ yields

$$
\mu^{*}=\frac{(1-\alpha)\left(\frac{\hat{\sigma}_{\eta}^{2}}{\hat{\lambda}}\right)^{\frac{1}{2}}-\hat{\sigma}_{\eta}^{2}-\hat{n}_{2}}{(1-\alpha) \hat{n}_{1}-\alpha\left(\hat{n}_{2}+\hat{\sigma}_{\eta}^{2}\right)}
$$

Since $\left\{\hat{n}_{1}, \hat{n}_{2}\right\}$ imply values for $\mu^{*}$ and $\hat{n}_{3}$, they are sufficient to characterize all equilibria. Assumption 1 ensures that agents acquire at least some information, so that $\hat{n}_{1}+\hat{n}_{2}>0$. Furthermore, if $\hat{n}_{1}+\hat{n}_{2}<\hat{n}$, the requirement that $\lambda_{1}(l) \geq 0$ and $\lambda_{2}(l) \geq 0$ ensures $\mu^{*} \in[0,1]$. Proposition 9 describes the necessary and sufficient conditions for this.

Proposition 9. Suppose that the cost of information is given by $c(\mu(l))=\lambda \hat{k}$. The the set of equilibria is characterized by $\left\{\hat{n}_{1}, \hat{n}_{2}\right\}$ that satisfy the one of the two sets of conditions below

- Case 1: Full Acquisition Only: $\hat{n} \leq(1-\alpha)\left(\frac{\hat{\sigma}_{\eta}^{2}}{\hat{\lambda}}\right)^{\frac{1}{2}}-\hat{\sigma}_{\eta}^{2}$

1. $\hat{n}_{1}=0$ and $\hat{n}_{2}=\hat{n}$

- Case 2: Multiple Equilibria: $\hat{n}>(1-\alpha)\left(\frac{\hat{\sigma}_{\eta}^{2}}{\hat{\lambda}}\right)^{\frac{1}{2}}-\hat{\sigma}_{\eta}^{2}$

- Case 2a

1. $(1-\alpha) \hat{n}_{1}<\alpha\left(\hat{n}_{2}+\hat{\sigma}_{\eta}^{2}\right)$

2. $\left(\frac{\hat{\sigma}_{\eta}^{2}}{\hat{\lambda}}\right)^{\frac{1}{2}} \geq \hat{\sigma}_{\eta}^{2}+\hat{n}_{1}+\hat{n}_{2}$

3. $(1-\alpha)\left(\frac{\hat{\sigma}_{\eta}^{2}}{\hat{\lambda}}\right)^{\frac{1}{2}} \leq \hat{\sigma}_{\eta}^{2}+\hat{n}_{2}$

- Case $2 b$

1. $(1-\alpha) \hat{n}_{1}>\alpha\left(\hat{n}_{2}+\hat{\sigma}_{\eta}^{2}\right)$ 

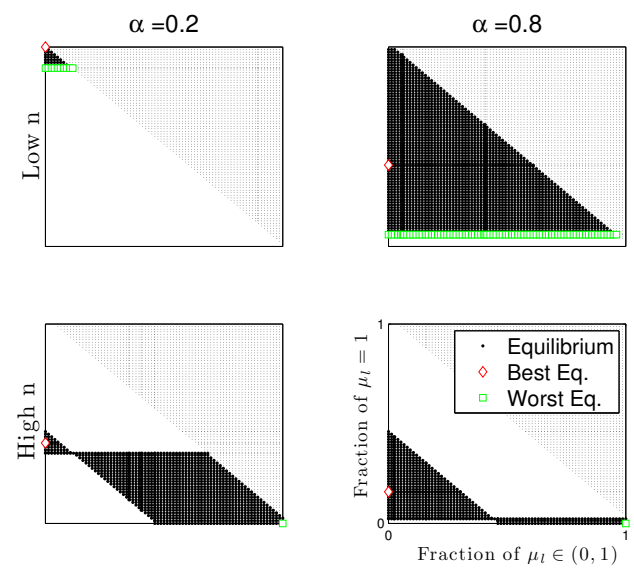

Figure 8: Multiple equilibria for different degrees of scope and strategic complementarity. The characteristics of the "best" and "worst" equilibria from the social planners perspective depend on the degree of scope.
2. $\left(\frac{\hat{\sigma}_{\eta}^{2}}{\hat{\lambda}}\right)^{\frac{1}{2}} \leq \hat{\sigma}_{\eta}^{2}+\hat{n}_{1}+\hat{n}_{2}$
3. $(1-\alpha)\left(\frac{\hat{\sigma}_{\eta}^{2}}{\hat{\lambda}}\right)^{\frac{1}{2}} \geq \hat{\sigma}_{\eta}^{2}+\hat{n}_{2}$

The two-by-two panel in figure 8 captures the range of multiple equilibria for different degrees of strategic complementarity, at different levels of scope. In the first row, with relatively low scope, equilibrium always entails some degree of perfectly directed search by agents. This result is closely related to the result in baseline model where, for low levels of scope, agents always choose to observe all signals. In this case, if agent $i$ can be assured that no others observe a particular signal $k$, then she may choose not to observe it as well. However, if others do observe that signal with positive probability, then she desires to do so as well, which increases the signal's informativeness, causing others to increase the probability with which they draw that signal, and so on. Once agents have acquired enough signals in this directed manner, however, this logic no longer bites, and agents may choose undirected search over some of the remaining signals. 
Higher strategic complementarities generally increase the scope for multiplicity. In the current model, this effect is apparent when scope is low. When scope is high, however, the consequences for multiplicity are more subtle. This contrast stems from the fact that, when complementarities are weak, agents are roughly indifferent to the degree of coordination in their information. As a result, agent $i$ is much less responsive in her own information choice to the degree to which other agents are direct their search. As a result, the set of equilibria under weak complementarity includes a range of information-search profiles that would be eliminated if agents had stronger strategic incentives. 